\title{
Fast Precise Point Positioning: A System to Provide Corrections for Single and Multi-frequency Navigation
}

\author{
A. Rovira-Garcia*, J.M. Juan*, J. Sanz*, G. González-Casado*, E. Bertran ${ }^{\dagger}$ \\ ${ }^{*}$ Research Group of Astronomy and Geomatics, ${ }^{\dagger}$ Dept. Signal Theory and Communications \\ Technical University of Catalonia, Barcelona, Spain
}

\begin{abstract}
Fast Precise Point Positioning (Fast-PPP) provides Global Navigation Satellite System corrections in real-time. Satellite orbits and clock corrections are shown to be accurate to a few centimeters and a few tenths of a nanosecond which, together with the determination of the fractional part of the ambiguities, enable global high-accuracy positioning with undifferenced Integer Ambiguity Resolution. The new global ionospheric model is shown to provide corrections accurate at the level of 1 Total Electron Content Unit over well-sounded areas and Differential Code Biases at the level of tenths of a nanosecond. These corrections are assessed with permanent receivers, treated as rovers, located at 100 to 800 kilometers from the reference stations of the ionospheric model. Fast-PPP achieves decimeter-level of accuracy after few minutes, several times faster than single- and dual-frequency ionospheric-free solutions, using a month of Global Positioning System data close to the last Solar Maximum and including equatorial rovers.
\end{abstract}

\section{INTRODUCTION}

The ionosphere plays an important role in satellite-based navigation, either in standard navigation with single-frequency mass-market receivers or in precise navigation with dualfrequency receivers. Single-frequency users correct the Global Navigation Satellite System (GNSS) measurements with the predictions provided by a broadcast ionospheric model. Thence, the accuracy of the ionospheric corrections is directly translated to the measurement modeling and, consequently, to the user solution.

Dual-frequency users typically use the Precise Point Positioning (PPP) technique to achieve high-accuracy positioning, i.e., at the sub-decimeter level in the kinematic mode [1], [2]. PPP is based on using more accurate satellite orbits and clocks, within a few centimeters, than those broadcast by the GNSS satellites. To compute these products, data from a global network of permanent receivers, e.g., the International GNSS Service (IGS) [3], must be accurately modeled and processed by a Central Processing Facility (CPF).

PPP users eliminate $99.9 \%$ of the ionospheric error in the GNSS signals with the dual-frequency ionospheric free combination of code and carrier-phase measurements. However, the noise of the raw GNSS measurements is amplified in this combination (a factor of 3 using the L1 and L2 signal bands of the Global Positioning System (GPS)). Moreover, carrierphase ambiguities are estimated as real numbers (floated ambiguities) instead of integers (fixed ambiguities). These are clear drawbacks compared with the classical Real Time Kinematics

This contribution is based on the best presentation award of the Session E4: High Precision GNSS Positioning in the 27th International Technical Meeting of The Satellite Division of the Institute of Navigation (ION GNSS+ 2014), Tampa, Florida, September 2014. Paper submitted December 2014, revised August 2015, January 2016, March 2016, May 2016, Accepted July 2016.
(RTK) technique [4], which works in differential positioning up to tens of kilometers from a reference receiver [5] canceling common errors and fractional biases by means of the Double Differences (DDs) of carrier-phase measurements.

As a result, PPP requires more time than RTK to achieve high-accuracy navigation. Indeed, the carrier-phase ambiguities are separated (i.e., decorrelated) from the other parameters in the navigation filter only when a significant change in the geometry of the satellites is observed by the receiver, which in case of floating ambiguities occurs after approximately one hour. This convergence time can be shorter using low-noise pseudoranges and/or using observations from more satellites (i.e., in a multi-constellation environment).

The accuracy of PPP can be further improved if the carrierphase ambiguities are fixed to their integer values, as in the aforementioned RTK technique. Two different techniques added to PPP the capability of Integer Ambiguity Resolution (IAR) in undifferenced mode. The first type defines different satellite clocks for code and carrier-phases [6], [7]. The second category uses only the usual satellite code clock and computes Single Differences (SDs) of carrier-phases between satellites [8], [9]. These IAR methods for PPP are compared in [10], [11] showing that once that the ambiguities are fixed, both approaches provide similar results.

The convergence time of PPP was shortened in [12], within a continental region (Europe), through the use of precise ionospheric corrections computed in an absolute mode (i.e., undifferenced). This last technique, known as Fast-PPP, was invented by the group of Astronomy and GEomatics (gAGE/UPC). The Fast-PPP technique using dual- and triplefrequency signals is protected since 2011 by several international patents [13] funded by the European Space Agency (ESA).

The present paper presents the consolidation of the Fast-PPP Global Ionospheric Map (GIM) introduced in [14]. The global 
coverage has been a requirement for the ESA-funded project named Ionospheric Conditions and Associated Scenarios for EGNOS (ICASES) [15], which has supported the best part of this activity. On a daily basis, reference ionospheric values (i.e., Slant Total Electron Contents (STECs)) are computed thanks to the precise modeling (at the centimeter level) of code and carrier-phase measurements done in the Fast-PPP CPF. These reference STECs have been used in [16] to characterize the accuracy of different ionospheric models for GNSS (i.e., Klobuchar [17], NeQuick [18] and IGS-GIM [19]) and Satellite Based Augmentation System (SBAS) (i.e., European Geostationary Navigation Overlay System (EGNOS) and Wide Area Augmentation System (WAAS)).

The current study presents not only the evaluation of the ionospheric determinations (as in [16]) but also the assessment of the accuracy of the complete set of Fast-PPP corrections: precise satellite orbits and clocks, fractional part of ambiguities and Differential Code Biases (DCBs). In particular, the accuracy of the Fast-PPP DCBs is compared to other IGS Analysis Centres (ACs). In this work we extend, in terms of duration and location, the assessment of the convergence time and the accuracy of the positioning errors achieved with the Fast-PPP corrections presented in [14]. Permanent IGS stations are navigated as rover receivers in kinematic mode, using one month of actual GPS data close to the last Solar Maximum, including equatorial locations.

The manuscript is divided as follows: the next section describes how the Fast-PPP CPF is implemented, and the routinely produced corrections are listed. The accuracy of the different corrections is assessed in the 3rd section, with special attention given to corrections related to ionospheric modeling. In the 4th section, using the accurate Fast-PPP corrections, the navigation improvement with respect to ionospheric-free solutions is reviewed. The discussion addresses not only users with a dual-frequency GNSS receiver but also rovers with single-frequency receivers. Moreover, navigation results are shown together with their protection levels, because the FastPPP CPF computes not only the corrections but also their confident bounds. Finally, the last section summarizes the results.

\section{FAST-PPP IMPLEMENTATION}

Fast-PPP corrections for high-accuracy navigation are computed in a unique CPF, with multi-frequency and multiconstellation capabilities [12]. The estimation of the different corrections is done with the three parallel Kalman filters shown in Fig. 1. The geodetic, the ionospheric, and the fast filter work with updating times of minutes and seconds, respectively.

The CPF is fed with GNSS data gathered by 3 different networks of permanent stations (see an example in Fig. 2). The first network (shown in black) includes all 172 receivers. Pseudorange and carrier-phase measurements, sampled every 30 seconds in this work, are modeled to the centimeter level in a pre-processing stage which includes cycle-slip detection and the computation of the ionospheric indicator named Along Arc TEC Rate (AATR) [20].

The pre-processed measurements are read by the geodetic filter to estimate every 5 minutes slow-varying parameters such

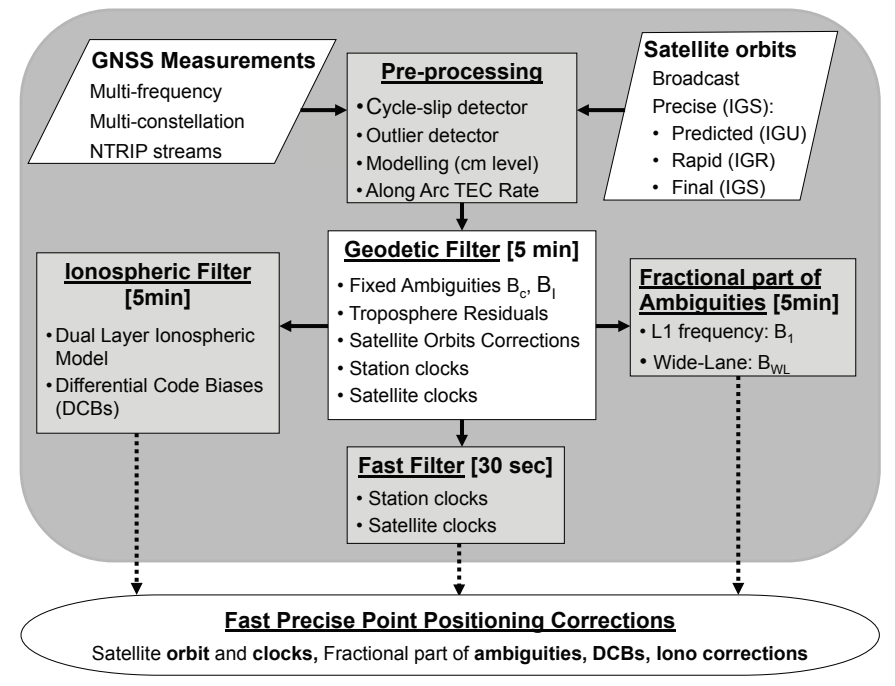

Fig. 1-Top-level design of the Central Processing Facility used in the Fast Precise Point Positioning technique.

as: the carrier-phase ambiguities, the troposphere residuals of the receiver network and the satellite orbit corrections to the input orbits (broadcast or precise). The main outputs of the geodetic filter are the carrier-phase measurements with fixed ambiguities, which are used by the other two filters (devoted to the estimation of the ionospheric model and satellite and receiver clocks).

The fast filter uses data from the second sub-network of 36 receivers shown in the blue in Fig. 2, to estimate the clocks of the satellites and stations of the network with a more frequent rate of 30 seconds. The fractional part of the ambiguities (for all receivers and satellites) is computed in a parallel module every 5 minutes.

Finally, a third ionospheric sub-network of 150 receivers (shown in red) is used to estimate the parameters of the Fast-PPP ionospheric model (described later in this work). In the current implementation, this sub-network covers the longitudinal range from -130 to 130 degrees and the latitudinal range from -90 to 90 degrees. The ionospheric model consists of a two-layer [21], irregular grid model, where the distances between the Ionospheric Grid Points (IGPs) are maintained approximately 250 and 500 kilometers in the first and second layers, respectively. The ionospheric structure is approximated with a first layer to represent the ionosphere and a second layer representing the plasmasphere (or upper ionosphere). The ionospheric filter updates the Total Electron Content (TEC) values of the IGPs and the satellite DCBs every 5 minutes, using the precise determination of the carrier-phase ambiguities done in the geodetic filter.

In addition to the three aforementioned networks, a subnetwork of permanent stations is navigated as rovers to assess the CPF products. These receivers are distributed worldwide (shown as green dots on the map), covering in particular 


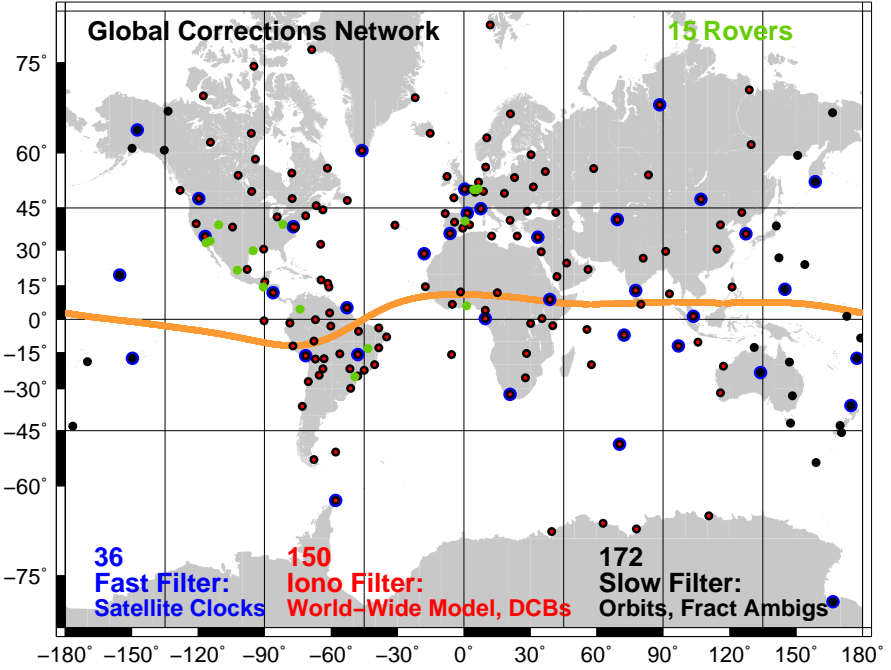

Fig. 2-Distribution of rovers (green) used in this study during DOYs 169-200 in 2014. All reference stations (black) are used to calculate orbit corrections, and fractional part of the ambiguities. The blue sub-network of stations is used to compute satellite clocks and the red sub-network to derive the ionospheric model. The geomagnetic equator is shown in orange.

the regions served by the current SBASs and their planned extensions which include equatorial regions. The distances from these rovers to the nearest ionospheric reference station range from 100 to 800 kilometers, which is more than one order of magnitude greater than the RTK or the Network RTK (NRTK) [22] baselines.

\section{FAST-PPP CPF CORRECTIONS ASSESSMENT}

As stated in the introduction, the Fast-PPP CPF has been automatically running daily since February 2014. This section presents an assessment of the quality of the GPS corrections computed by the Fast-PPP CPF from Day Of Year (DOY) 169 to DOY 200 in 2014 (i.e., a month of data). In order to characterize the ionospheric conditions of the assessment, two indicators are presented in Fig. 3: i) the solar flux (top) indicates that the selected period (dots) is close to the Solar Cycle maximum, ii) the hourly $D_{s t}$ index (bottom) [23] indicates that the geomagnetic activity is not perturbed (because of the absence of geomagnetic storm conditions, that is $\left.D_{s t}>-50 n T[24]\right)$.

\section{Fast-PPP Satellite Orbits}

The Fast-PPP CPF orbit correction module can work from broadcast or IGS orbits, as shown in Fig. 1. Because the ICASES project (which, as aforementioned, supported this study) targets periods of high ionospheric activity with STEC variations up to several meters in 30 seconds, classical cycle slip detectors [25] based on a smooth ionosphere cannot be used. Indeed, special cycle slip detectors have been developed
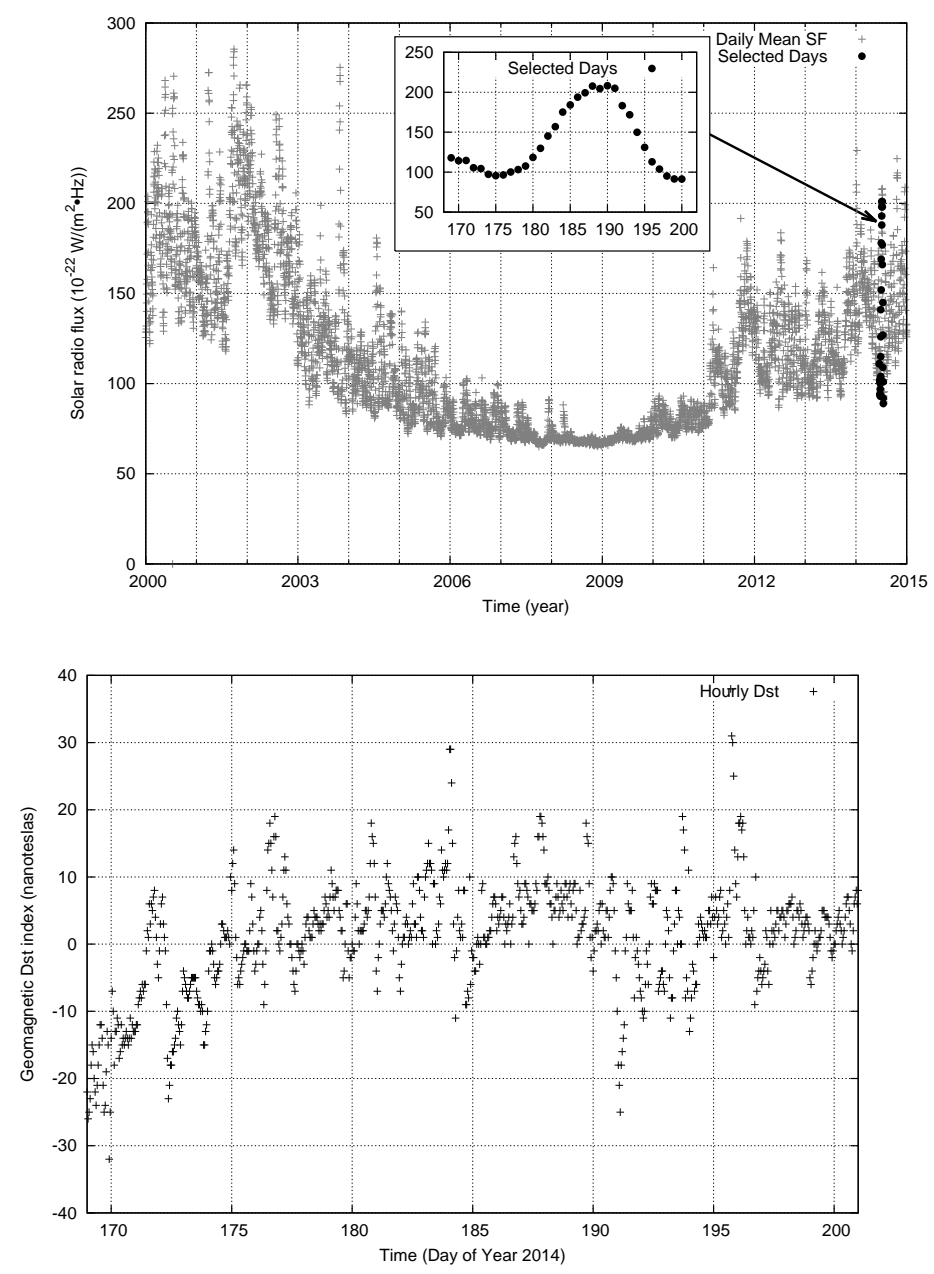

Fig. 3-Top - Radio solar flux (pluses) since 2000 with a zoom (dots) for the selected period (DOYs 169-200 in 2014). Bottom - The geomagnetic disturbance index, Dst (pluses) over the period of interest.

that require accurate orbits and clocks, such as, the postprocess IGS Rapid (IGR) products [26]. In this regard, satellite orbits and clocks sampled every 15 and 5 minutes, respectively, are used to build the pre-fit measurements every 30 seconds without flagging false detections. For this reason, in the ICASES project, the Fast-PPP CPF does not estimate orbit corrections. However, to show that this study does not take advantage of using IGR orbits, the first week has also been processed using the predicted part of the IGS Ultra-Rapid (IGU) orbits (the mode for real-time navigation). These IGU orbits are adjusted in the Fast-PPP CPF using code and carrierphase GNSS measurements. As it is shown in the section assessing the navigation results, no significant differences are observed regarding the usage of IGU or IGR products.

In Fig. 4, the hourly Root Mean Square (RMS) of the 3D 


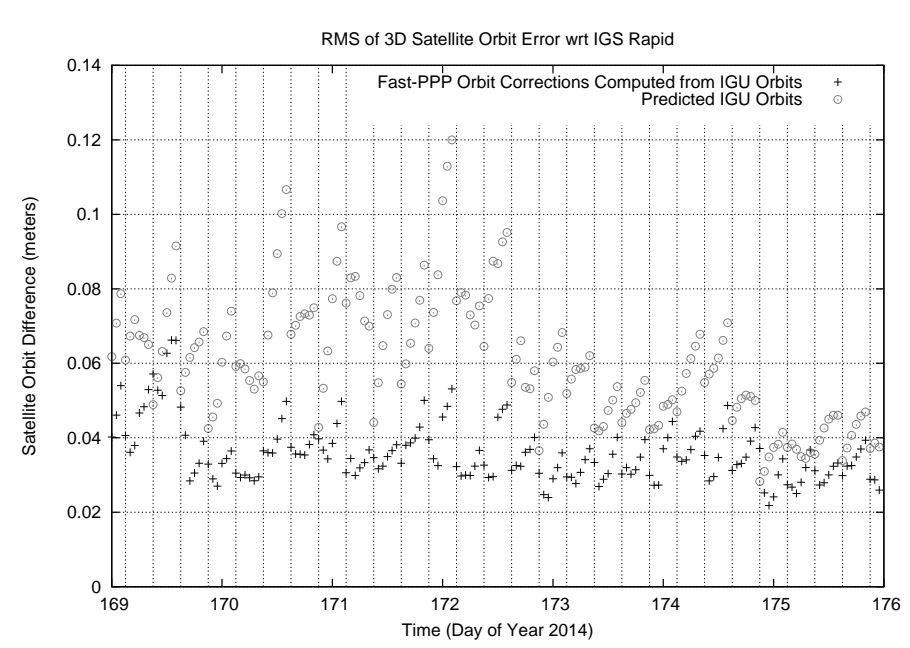

Fig. 4-Hourly RMS of the 3D orbit difference with respect to the IGS Rapid (IGR) orbits for DOYs 169-176 in 2014. FastPPP CPF computes real-time orbit corrections (pluses) to the predicted IGS Ultra-Rapid (IGU) orbits (circles).

TABLE I. RMS OF THE DIFFERENCE BETWEEN THE SATELLITE ORBIT AND CLOCK DETERMINATIONS WITH RESPECT TO THE IGS RAPID PRODUCTS.

\begin{tabular}{c|c||c|c}
\hline \multicolumn{2}{c||}{ 3D Orbit Error } & \multicolumn{2}{c}{ Satellite Clocks } \\
\hline \hline Fast-PPP & IGU & Fast-PPP & Fast-PPP \\
Correct IGU & Raw & Correct IGU & Raw IGR \\
$3.7 \mathrm{~cm}$ & $6.3 \mathrm{~cm}$ & $0.17 \mathrm{~ns}$ & $0.20 \mathrm{~ns}$ \\
\hline
\end{tabular}

orbit differences between the IGU orbits and the IGR orbits is shown using circles. The degradation of the predicted IGU orbits is evident as they become "older". In contrast, the IGU orbits corrected by the CPF in real-time (pluses), mitigate the orbit degradation until a new orbit prediction set is delivered every 6 hours (corresponding to the time tics in Fig. 4). In this regard, the RMS of the orbit error is 3.7 centimeters, in line with other centers that compute satellite orbits in the IGS Real-Time Pilot Project (IGS-RTPP) [27].

\section{Fast-PPP Satellite Clocks}

Unlike with satellite orbits, the Fast-PPP CPF always estimates satellite clocks regardless of the orbit source. The assessment of the satellite clock corrections product is done by directly comparing with the IGR clocks. In Fig. 5, it is shown the hourly RMS of the difference between the IGR clocks and the Fast-PPP clocks when the Fast-PPP CPF starts from the predicted part of the IGU orbits (grey crosses) and from the IGR orbits (black pluses). It can be observed that both orbit sources produce similar satellite clock corrections, which are even slightly better when the orbits are corrected. In both cases, the RMS is typically below 0.2 nanoseconds (see Table I), which is in line with the performance data for the clock product obtained by other analysis centers of the aforementioned IGS-RTPP [27].

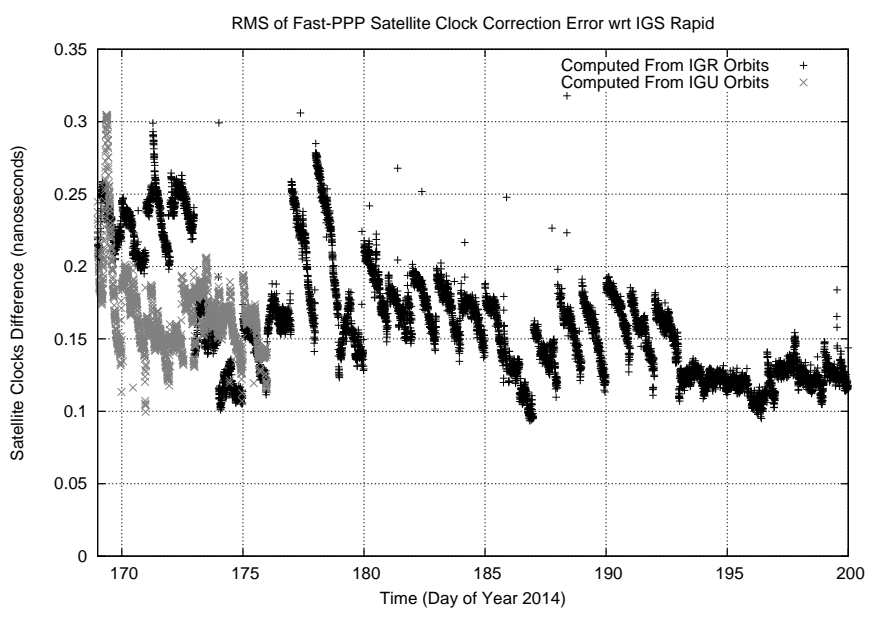

Fig. 5-Hourly RMS of the difference between the real-time satellite clock corrections with respect to the IGS Rapid clocks for DOYs 169-200 in 2014. Fast-PPP CPF can compute the satellite clocks either from the post-process IGS Rapid (IGR) orbits (black) or from the predicted IGS Ultra-Rapid (IGU) orbits corrected by the Fast-PPP CPF (grey).

\section{Fractional part of the ambiguities}

PPP users can enhance their positioning accuracy by fixing the carrier-phase ambiguities to integer values. This can be done in undifferenced mode and on a worldwide basis, because the Fast-PPP CPF is capable of estimating the satellite fractional part of the ambiguities [6]-[9]. Although there is not a standard product available for use as reference and the value depends on the reference taken in the $\mathrm{CPF}$, the agreement between the independent estimations (i.e., consecutive days) can be assessed.

Figure 6 shows the fractional ambiguities of three representative satellites for the L1 band, (B1, top), and the WideLane combination, (BW, bottom), computed using both the IGR orbit (post-process mode) and the corrected IGU orbits (real-time mode). From these plots, the following results can be inferred: i) the fractional parts of the ambiguities computed by the Fast-PPP CPF are parameters with a slow variation, and can thus be broadcast to users with a large time update (e.g., every 5 minutes in this implementation), ii) the fractional B1 ambiguity daily trend is related with the correlations with other parameters estimated in the geodetic filter (i.e., satellite orbits and clocks and troposphere), and presents discontinuities, on a day-to-day basis, typically smaller than a 0.1 cycle (2 centimeters) when IGU orbits are corrected, iii) the consistency of B1 is slightly degraded when the IGR orbits are used (disabling the orbit correction module), indicating that some residual orbit error is transferred to the ambiguity estimation when the IGR orbits are used, instead of the usual module implemented in the $\mathrm{CPF}$, and iv) the repeatability of the $\mathrm{BW}$ fractional ambiguity does not depend on the orbit source.

At the user level, the satellite fractional part corresponds to the term $\delta B^{j}$ in equation (1) for an ambiguity, $B_{i}^{j}$, for 
a particular frequency (and corresponding wavelength, $\lambda$ ) between a receiver, $i$, and a satellite, $j$. The receiver fractional part, $\delta B_{i}$, is common to all satellites sharing that frequency.

$$
B_{i}^{j}=\lambda N_{i}^{j}+\delta B_{i}+\delta B^{j}
$$

The ambiguity fixing strategy is given by equation (2), in which the receiver fractional part is eliminated by differencing with respect to a reference satellite, $B_{i}^{0}$ (usually with the highest elevation angle). The satellite fractional parts are removed with the CPF corrections, $\left(\delta B_{i, \text { cor }}^{j}, \delta B_{i, c o r}^{0}\right)$.

$$
\left(B_{i}^{j}-\delta B_{i, c o r}^{j}\right)-\left(B_{i}^{0}-\delta B_{i, c o r}^{0}\right)=\lambda\left(N_{i}^{j}-N_{i}^{0}\right)
$$

Three simultaneous conditions must be fulfilled before the Fast-PPP user fixes the ambiguities: (i) the formal error of the floated ambiguities $\left(B_{i}^{j}, B_{i}^{0}\right)$ must be under a certain threshold (e.g., 0.8 meters), (ii) the left hand side of equation (2) divided by $\lambda$ must be close enough to an integer (e.g., 0.15 cycles), (iii) a minimum number of epochs (e.g., 300 seconds) since the last cycle-slip of the arc are also required. When these tests are successful, the term $\left(N_{i}^{j}-N_{i}^{0}\right)$ can be fixed to an integer value. Therefore, the constraint of equation (2) is added to the navigation filter with a great confidence (low sigma).

\section{FAST-PPP IONOSPHERE}

The core of the Fast-PPP technique is the real-time determination of the ionospheric delay present in the GNSS signals, i.e., the STEC. Indeed, once the satellite orbit and clocks are determined with errors at the level of few centimeters, the accuracy of the ionospheric delay modeling should be better than 1 Total Electron Content Unit (TECU), which corresponds to 16 centimeters in L1. Otherwise, errors in the ionospheric corrections larger than few tenths of centimeters would shatter the accuracy of the geodesy estimates in the position domain.

The Fast-PPP ionospheric model is computed from the output of the geodetic filter. The ionospheric filter uses as input the geometry-free combination of carrier-phase measurements ( $L I=L 1-L 2[25])$, corrected from the non dispersive effects, which are estimated in the geodetic filter. In particular, the ionospheric combination is corrected from its fixed carrierphase ambiguity $(B I=B 1-B 2)$. In this way, for a receiver, $i$, and the satellite, $j$ the unambiguous $L I_{i}^{j}-B I_{i}^{j}$ is modeled as:

$$
L I_{i}^{j}-B I_{i}^{j}=S T E C_{i}^{j}+D C B_{i}-D C B^{j}
$$

Then, the left hand side of equation (3) can be treated as a geometry-free combination of carrier-phase data acting as pseudorange measurements $(P I=P 2-P 1[25])$ with reduced noise. In the right hand side of equation (3), the satellite and receiver DCBs between $P 2$ and $P 1, D C B^{j}$ and $D C B_{i}$ appear together with the ionospheric delay, $S T E C_{i}^{j}$.

In order to distinguish between the DCB of the receivers and the satellites in equation (3), a reference DCB value must be selected. This reference can be the value of a particular satellite or receiver DCB or, as it is usually done in IGS, imposing a zero mean condition to the average of the satellite DCBs.
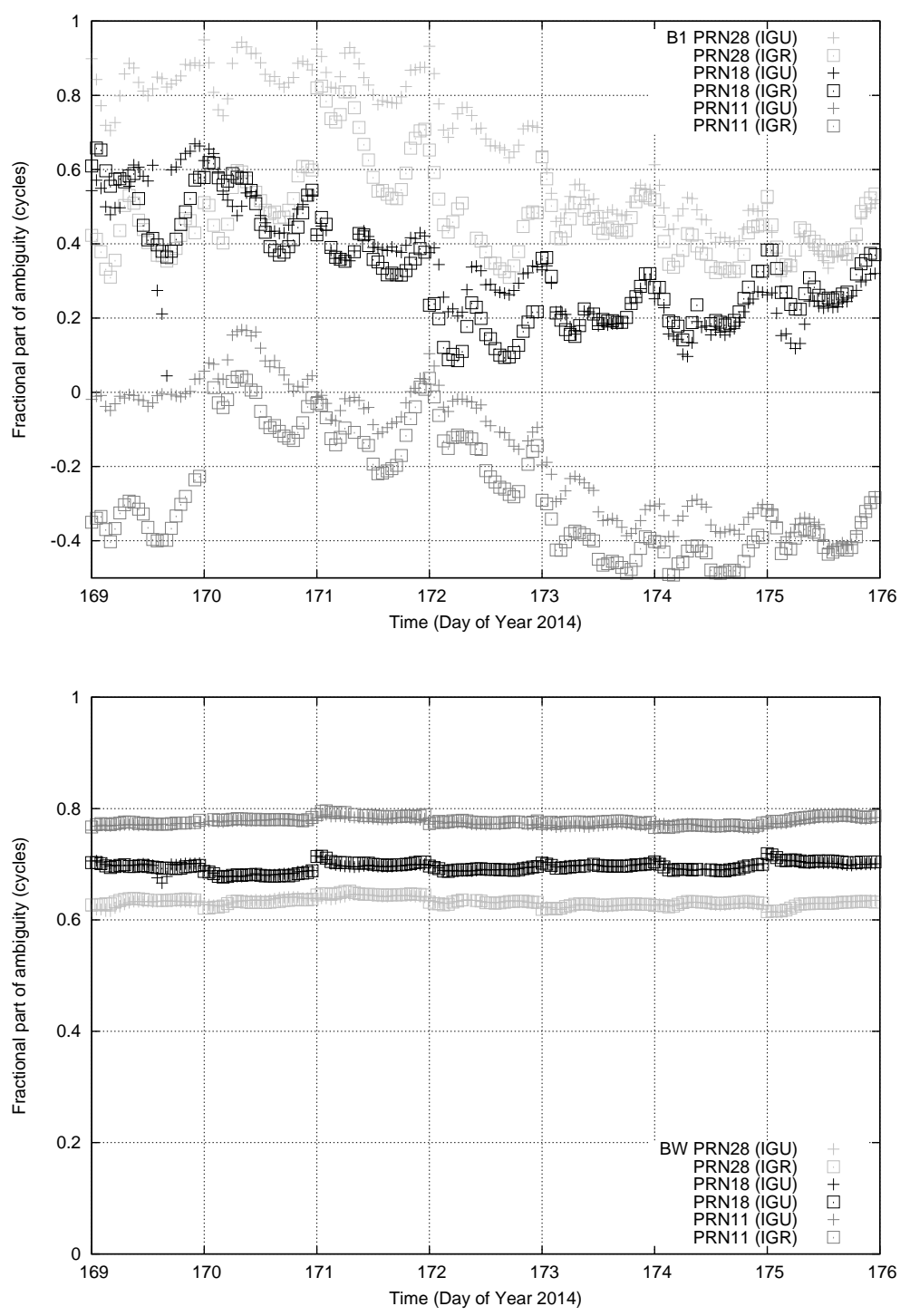

Fig. 6-Satellite fractional part of the ambiguities in units of cycles. The BI (top) and the Wide-Lane (BW) combination (bottom) are computed using two type of IGS orbit sources, IGR (squares) and IGU (pluses).

However, the election of the DCB reference has no impact in the accuracy of the ionospheric or DCB estimates.

The STEC is expressed as a linear combination of the Vertical Total Electron Content (VTEC) following equation (4):

$$
S T E C_{i}^{j}=\sum \alpha_{k} \cdot V T E C_{k}
$$

where $\alpha_{k}$ is a geometric factor that includes the obliquity factor and the geometric interpolation from the Ionospheric Pierce Points (IPPs) to the surrounding 4 IGPs in the first (main ionosphere) and second (plasmasphere) layer, totaling 8 IGPs per ray. The IGPs are distributed using a constant 250 


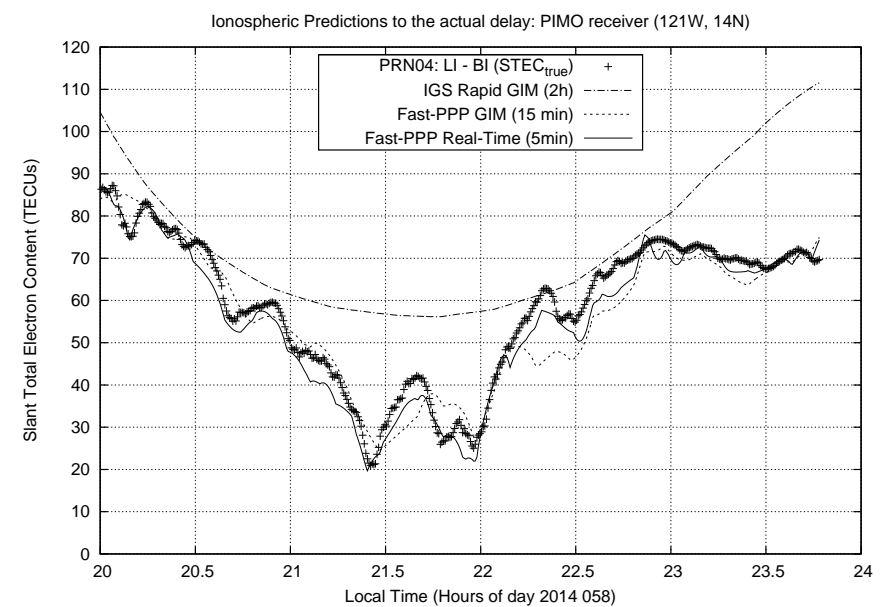

Fig. 7-The unambiguous and unbiased STEC (pluses) every 30 seconds for PRN 23 at the IGS receiver PIMO $\left(121^{\circ} \mathrm{W} 14^{\circ} \mathrm{N}\right)$ is predicted by; the 2-h GIM from IGS (dash-dotted line), the 15minute Fast-PPP GIM delivered to the ICASES project (dashed line), and the real-time 5-minute Fast-PPP ionosphere using two layers (solid line).

and 500 kilometers spatial resolution in MOdified DIP latitude (MODIP) [28] and longitude, respectively. The ionospheric filter of the Fast-PPP CPF estimates every 5 minutes, the vertical ionospheric delays, $V T E C_{k}$, separated from the DCBs [29]. Figure 7 depicts an example of the STEC computed by the Fast-PPP real time model (solid line) from the unambiguous $(L I-B I)$ shown with pluses, together with some GIM determinations latter explained.

\section{Ionospheric model assessment}

The assessment of the accuracy of any ionospheric model for GNSS navigation is an open issue because, unlike of satellite orbits and clocks, there is no ionospheric reference model with the required accuracy (i.e., better than 1 TECU). For this reason, a test has been developed [16] using the unambiguous determinations as a reference value (i.e., the $S T E C_{t r u e}$ ) computed by the CPF, according to the equation (5):

$$
S T E C_{t r u e, i}^{j}=L I_{i}^{j}-B I_{i}^{j}-\left(D C B_{i}-D C B^{j}\right)
$$

The $S T E C_{t r u e, i}^{j}$ values derived from equation (5) represent the true STEC values, except for inaccuracies in the DCB estimates (few centimeters, later shown in Fig. 9) and the fractional ambiguity in the $B I$ (few centimeters, already shown in Fig. 6). Then, the term $S T E C_{\text {true }}$ is introduced regarding to the ionospheric test proposed next, in which the difference between the prediction of any ionospheric model and the reference is separated as hardware delays in equation (6) with a constant for the receiver $K_{i}$ plus a constant for the satellite $K^{j}$ :

$$
S T E C_{\text {model }, i}^{j}-S T E C_{\text {true }, i}^{j}=K_{i}+K^{j}
$$

The Least Squares (LS) process is used to estimate $K_{i}$ and $K^{j}$ on the right side of equation (6). By estimating the $K^{\prime} s$, the results of the test are not affected by inaccuracies in the DCB and $B I$, as these errors are absorbed in the constant values. The post-fit residuals of this adjustment provide a metric for assessing the realism of the STECs predicted by the ionospheric model. Any common bias in the STECs affecting both satellites and/or receivers will not affect the test results, because the bias is absorbed into the receiver and satellite constants. In this sense, this test assesses the ionospheric predictions of any model on a global scale, because $K_{i}$ and $K^{j}$ are set as common constant parameters for the entire network of receivers (and satellites) over the time interval (typically batches of 24 hours). Numerically, using data sampled every 30 seconds from the 150 stations of the ionospheric network example shown in Fig. 2 and an average of 8 satellites in view per station, each day approximately 3.5 million STECs are fit to 182 parameters $\left(150 K_{\text {sta }}+32 K^{\text {sat }}\right)$.

The post-fit residuals after solving equation (6) are shown in Fig. 8 for different ionospheric models at the receivers shown in red in Fig. 2. In the case of Fast-PPP, together with the ionospheric model parameters (VTEC at the IGPs and DCBs), the ionospheric filter computes the STEC prediction for reference receivers to have an idea about the quality of the adjustment. However, in order to reduce the computational burden, the predictions are only computed for a subset of 40 stations. The Fast-PPP real-time estimates at such subset of receivers (crosses) present the best agreement with respect to unambiguous carrier-phase measurements, with residuals at the level of few tenths of 1 TECU. Notice that such test results reflect only the losses occurring in the process of fitting the STEC delays to vertical values at the IGPs of the reference stations used by the ionospheric filter to derive the Fast-PPP model. As it is later discussed, the error of the Fast-PPP model at independent stations (i.e., at the rovers receivers) can be several times larger.

The Fast-PPP ionospheric model is limited to the regions where data are available in real time, covering the vicinity of permanent receivers; there are no estimations for the IGP on poor sounded areas (i.e., the oceans). In order to generate the Fast-PPP GIM, it is necessary to include some smoothing constraints (in post-process) that degrade the well-sounded (continental) IGPs obtained in the real-time model. An example of the STECs obtained with the smoothed Fast-PPP GIM is depicted in Fig. 7 using a dashed line. The correspondent post-fit residuals for the Fast-PPP GIM are depicted in Fig. 8 with circles, reaching about 1 TECU.

The Rapid or Final Ionospheric Products from IGS [19] are commonly used to model the TEC, see for instance the dash-dotted line in Fig. 7, with a nominal accuracy of 2-8 TECU in vertical (i.e., not slant) [26]. The residuals shown in Fig. 8 with plus signs correspond to the Rapid IGS GIM, being several times greater than the Fast-PPP GIM. Both GIM post-fit residuals are computed for the 150 stations shown in red in Fig. 2 and exhibit a daily periodicity following the local 
time (Fig. 8, top plot) and a monthly variation related with the solar radio flux (see zoom of top plot in Fig. 3).

It must be noted that the IGS GIM, the Fast-PPP GIM and the Fast-PPP real-time model are updated every 2 hours, every 15 minutes, and every 5 minutes, respectively. Nevertheless, although the lower time updates contribute to reduce the error, the key factor improving the accuracy is the processing strategy of Fast-PPP as a whole:

First, the use of a dual-layer strategy to separate the main ionosphere and the plasmasphere. Each component has a different height and dynamic evolution. As it is shown in [14], the errors of the Fast-PPP real-time model doubled in the equator when the number of layers was reduced to one.

The two-layer description of the Fast-PPP model has shown to be able to distinguish [30] the ionospheric delay at altitudes beyond a thousand of kilometers (plasmasphere) from the delays occurring in the F2 layer (ionosphere). Specifically, it was shown a qualitative level of agreement between the Fast-PPP GIM (derived with ground measurements) and independent TEC measurements from Radio Occultations (ROs) of Low Earth Orbit (LEO) satellites.

Second, the geometry of the model taking into account the MODIP [28] to refine the grid at low latitudes. Third, the use of fixed carrier-phase ambiguities as constrains in the ionospheric filter. The differences between processing strategies are more noticeable when the RMS test results are compared on a latitude basis (Fig. 8, bottom plot), specially in the equatorial region.

\section{Satellite Differential Code Biases}

The determination of the satellite DCBs between the $P 2$ and $P 1$ pseudorange measurements ${ }^{1}$ is a key factor for users applying ionospheric corrections; otherwise, those biases would worsen the user positioning (particularly for single-frequency users). The ionospheric filter estimates the DCBs (treated as constant parameters within each daily batch) every five minutes in real-time by using unambiguous carrier-phase measurements and the Fast-PPP ionospheric model (see equation (3)). DCB estimations are independent from day to day, because the DCBs are not constrained to any particular value. In order to allow the convergence of the estimation of STECs and DCBs, the Fast-PPP CPF uses the last 10 hours of the previous day before providing corrections in real time to the rover receivers.

Usually, the determination of the DCB depends on the pseudorange noise, which is approximately two orders of magnitude greater than the carrier-phase measurement noise. However, all observations from the same satellite (or receiver) share the same DCB, which implies a high redundancy of observations. Two factors (related to geometry and time evolution) are of great importance to allow the ionospheric filter to separate the constant contribution (DCB) from the variable part (STEC) in equation (3) [29]. First, every satellite is observed by mixed rays from a global distribution of stations with many different elevation angles (i.e., different $\alpha_{k}$ coefficient

\footnotetext{
${ }^{1}$ The bias between $C 1-P 1$ is corrected when pseudoranges are modeled using the monthly determination from Centre for Orbit Determination in Europe (CODE).
}
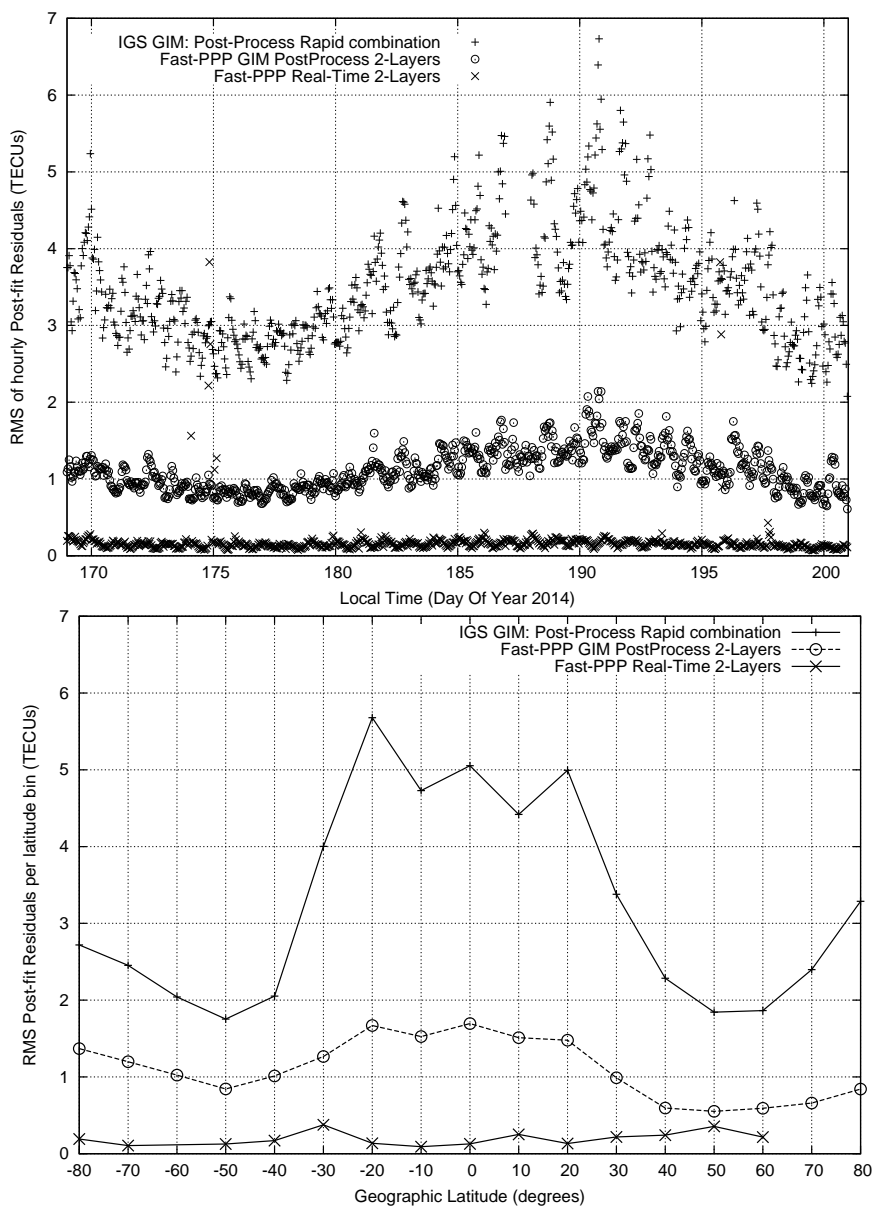

Fig. 8-Results of the consistency test between different ionospheric estimates; 2-h GIM IGS combined Rapid product (pluses), a 15-minute GIM delivered to ICASES project (circles), and the real-time 5-minute Fast-PPP ionosphere using two layers (crosses). The horizontal axis is the local time in the top plot and latitude in the bottom plot. The RMS of the post-fit residuals are computed every hour in the top plot and represented with latitude bins in the bottom plot for the entire period.

for the mapping in equation (4)). Second, the observations for each satellite occur simultaneously (same Universal Time (UT)) from receivers placed at different Local Times (LTs) and latitudes, which means that very different STECs (with different mappings) are sharing the same satellite DCB.

The bottom plot of Fig. 9 depicts the DCBs calculated by the Fast-PPP CPF every 5 minutes for a month of data starting in June 2014 (DOYs 169-200). The observed DCB values differ from the IGR product (not shown) in few tenths of a nanosecond, which is a typical difference between different AC of IGS [19]. Some discontinuities common to all satellites are observed, as a result of having imposed to zero the mean of the satellites DCB when solving equation (3). The top plot of Fig. 9 shows that a change in the reference DCB value (for 


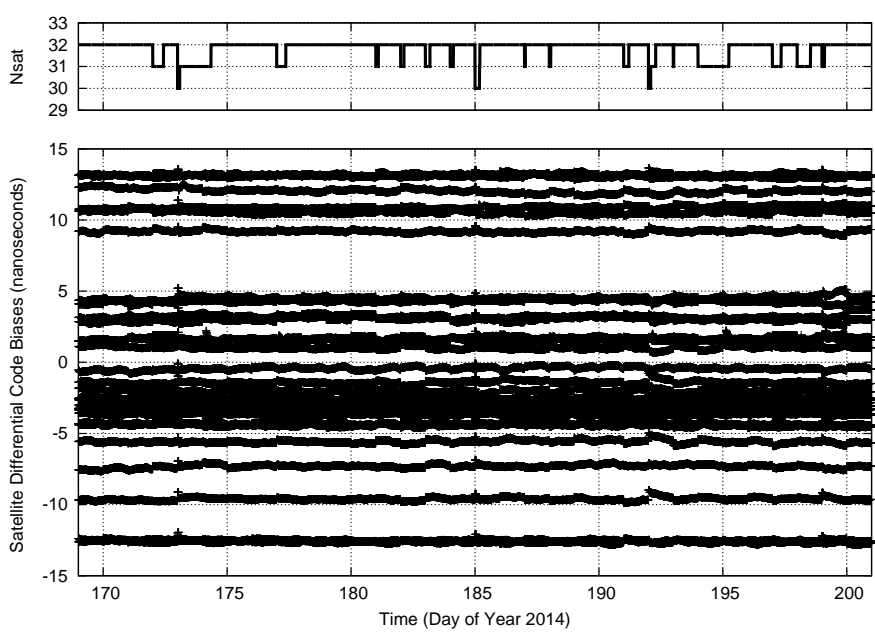

Fig. 9-Fast-PPP Satellite DCB estimation (bottom plot) for a total number of satellites (top plot) every 5 minutes.

instance, a satellite that appears or disappears during the day) causes a common bias to all the satellite DCBs. However, this bias common to all satellites does not worsen the positioning accuracy of Fast-PPP users, as it is absorbed in the DCB estimation of the rover receivers.

Because DCBs are estimated independently each day, at the same time that the ionospheric model, the stability of the DCBs from day to day is an additional indicator of how correctly the ionospheric delay has been determined by means of the ionospheric model. Indeed, the accuracy of the Fast-PPP ionospheric estimates has been used to detect and to analyze DCB anomalies (jumps and drifts) at the receiver and satellite level [31].

Figure 10 depicts the standard deviation (for all satellites) of a number of DCBs estimates for the 31 days period. It includes determinations from different ACs of IGS: Technical University of Catalonia (UPC), European Space Operations Centre (ESOC) and CODE. Notice that IGS products only provide a daily value in post-process. Then, in addition to the standard deviation of the Fast-PPP determinations computed in real-time every 5 minutes (shown in the histogram), the final real-time estimation of each day is selected to compute a daily standard deviation (shown with a line with pluses signs).

The average standard deviation of the Fast-PPP 5 minute estimation is 0.10 nanoseconds, while the daily estimation is 0.07 nanoseconds. This result indicates that to consider only a daily DCB partially mitigates the ionospheric missmodeling absorbed in the DCB estimation, i.e., this error propagation is sampled with the standard deviation computed every 5 minutes. Regarding to the IGS ACs results, the average standard deviation of the DCBs computed by UPC (crosses) and ESOC (squares) are both at the level of 0.14 nanoseconds. The lowest standard deviation is observed in the CODE DCBs, which is 0.06 nanoseconds. Nevertheless, it must be noticed that CODE DCB is a three-day solution, which should be smoother than one-day solution.

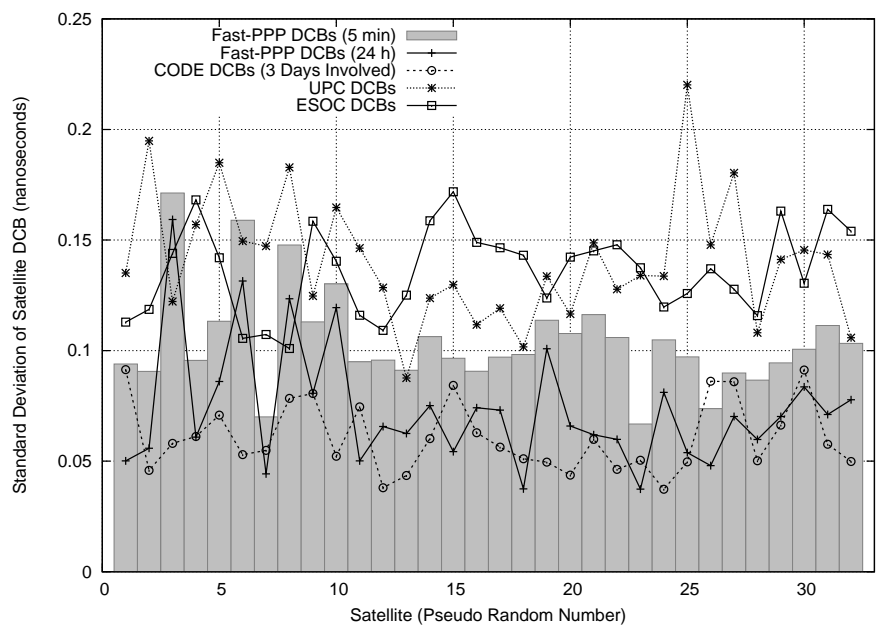

Fig. 10-Fast-PPP Satellite DCB estimation every 5 minutes (top plot) and its standard deviation (bottom plot, boxes) for different satellites identified by the PRN. The bottom plot includes the standard deviation of the satellite DCBs the computed every 24 hours by Fast-PPP and different ACs belonging to IGS.

\section{FAST-PPP NAVIGATION}

The Fast-PPP user navigation strategy [14] keeps code and carrier-phase measurements (L1, L2, P1, P2) separate from the CPF corrections. These corrections are included in the navigation filter as additional equations with their associated confidence bounds (sigma). This is similar to the weighted ionospheric approach described in [32], but in absolute (i.e., undifferenced) mode. In this sense, not only the values of the corrections are relevant, but also their confidence bounds. Less accurate corrections as a result of a poor estimation, network outage or ionospheric events do not worsen the user positioning because they are bounded by a large sigma.

In this section, the user positioning achieved by the current implementation of the Fast-PPP technique, is examined. First, it is shown how the accurate geodesy corrections (satellite orbits and clocks), computed by the $\mathrm{CPF}$, enable a highly accurate navigation for single- and multi-frequency users, regardless of the orbit source (IGR or IGU). Subsequently, the realtime Fast-PPP ionospheric corrections are proved to shorten the convergence time to achieve such precise positioning. The improvement is measured with respect to the single- and dualfrequency ionospheric-free navigation techniques, respectively; Group and Phase Ionospheric Calibration (GRAPHIC) [33] and PPP [2].

Notice that the modeling accuracy of GRAPHIC and PPP is at the centimeter level and do not present any bias related to the ionosphere. This allows us to use these combinations as reference solutions when ionospheric corrections are used. The drawback of such ionospheric-free solutions (singleand dual-frequency) is that the carrier-phase ambiguities are mainly estimated with the pseudorange measurements. The code noise slows the filter convergence to achieve decimeterlevel of accuracy, usually lasting one hour for PPP and few 


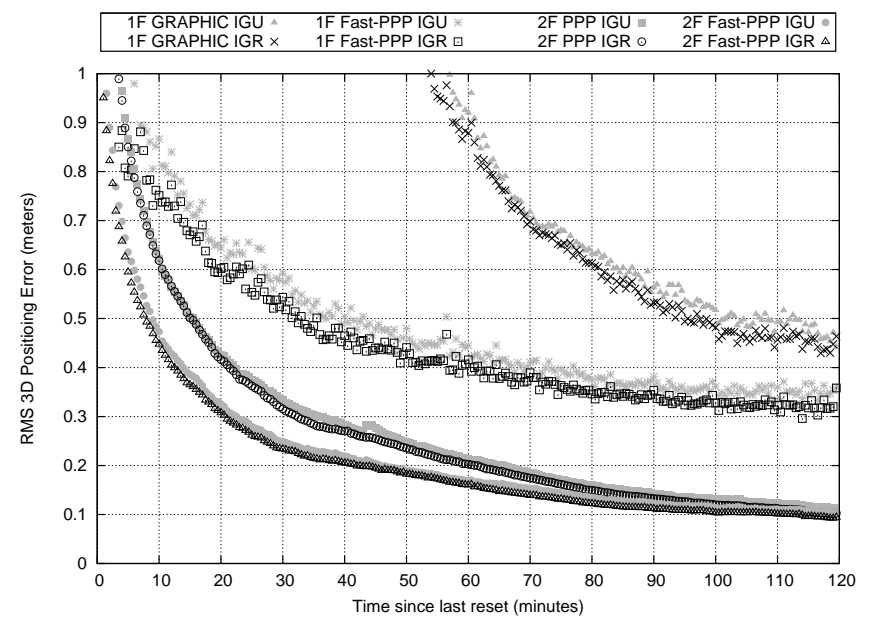

Fig. 11-RMS of the 3D error as function of the elapsed time since the receiver is reset (every 2 hours). Curves involve all of the rovers present in DOYs 169-176 in 2014 for two IGS orbits sources: predicted IGU (black) and post-process Rapid IGR (black).

hours for GRAPHIC. To add ionospheric corrections to the navigation filter, as additional information, allows the user to reduce the convergence time. Moreover, if the corrections are unbiased such as Fast-PPP, the final solution converges to the ionospheric-free accuracy much earlier. This is not the case when using ionospheric corrections that present biases.

Finally, the Stanford plots [34] are used to assess how the Signal In Space (SIS) confidence bounds are transferred to the user domain, showing the internal consistency of the parameterization and hypothesis assumed in the Fast-PPP navigation, achieving adequate protection levels.

\section{Orbit Corrections Assessment}

The impact on the position domain of using the FastPPP corrections computed from the post-processed IGR orbits without correcting them or correcting the predicted IGU orbits is assessed in Fig. 11. The figure shows the merged 3D RMS error of all rover solutions for one week (DOYs 169-176 in 2014), resetting the filter state every two hours. The black curves correspond to the navigation achieved using IGU orbits, and the gray curves correspond to the navigation using IGR orbits. It can be observed that both orbit sources provide similar results for the ionosphere-free solutions (GRAPHIC, PPP) and the ionospheric (Fast-PPP) solutions in the singleand dual-frequency navigation modes. Therefore, it is also confirmed at the user domain that there is no added benefit to compute the Fast-PPP corrections using the post-process IGR Rapid orbits from IGS, with regard to the use of IGU orbits.

\section{Convergence Assessment}

The reduction of the convergence time of the user positioning by means of ionospheric modeling is observed after a user cold start or a cycle slip. The carrier-phase ambiguity estimation is shortened by the Fast-PPP ionosphere, because it is absolute (i.e., unambiguous) and several times more precise than the code measurements. Otherwise, in PPP or GRAPHIC, ambiguities are mostly estimated with the noisy (although unambiguous) code. Fast-PPP users that are fixing the carrierphase ambiguities also reduce the Time To First Fix (TTFF). The relation between the ionospheric performance of Fig. 12 and the convergence of the rovers in Fig. 13 is explained below.

The efficiency of the ionospheric corrections in the navigation filter decreases with the error of the ionospheric model. As already commented, Fig. 8 presented the post-fit residuals at the reference stations, sampling only the error of the ionospheric model of representing unambiguous STECs by means of a grid. Now it is time to analyze a second source of error in the ionospheric corrections, that is the interpolation from the stations that are used to derive the ionospheric model to the user location. Such contribution to the total error depends on: (i) the ionospheric activity shown in Fig. 3, (ii) the distribution and distance to the reference stations, and (iii) the user latitude.

Figure 12 presents the ionospheric error of the Fast-PPP real-time model at different rover locations and distances during the maximum solar flux of the period of time covered by the data set. To obtain the error, we have processed twice the ionospheric values, firstly considering the rovers as a reference station to compute their $S T E C_{\text {true }}(L I-B I)$. Secondly, the ionospheric model is re-computed excluding the rovers from the processing, thence the STEC for the rovers is computed using only the data from the reference stations. The figure depicts the RMS of the difference between the predictions of the Fast-PPP real-time model (5 minutes) and the reference values at the rover sites. Notice that the STEC errors for rovers in well-sounded areas are comparable to the Fast-PPP GIM, rather than to the Fast-PPP real-time model at the reference stations. In contrast, for rovers in poor-sounded areas the errors are clearly greater than 1 TECU.

It can be observed that in middle latitudes the interpolation error is small: up to 100 kilometers (eijs station, filled dots) the error is at the same level as a reference station $(0.5$ TECUs). The degradation increases with the distance, but the error remains at the 1 TECU level (i.e., the Fast-PPP GIM) at distances of a few hundred kilometers (ebre station, squares). At low latitudes, the degradation is more noticeable with respect to distance (ufpr station, pluses); at 140 kilometers, the error already reaches the 1 TECU level. The error at rover ineg (crosses) reaches several TECUs, not only because of its equatorial latitude but also because the lack of nearby stations. According to Table II and Fig. 2, ineg is located in the Mexican Pacific coast within 460 and 1400 kilometers of the two nearest reference stations. The isolation of this rover significantly contributes to the measured ionospheric errors.

The accuracy of the ionospheric model is translated to single- and dual-frequency positioning as shown in Fig. 13, where the Horizontal Positioning Error (HPE) and Vertical Positioning Error (VPE) together with their 3D formal error are shown in the left, center and right column, respectively. The plots merge 31 days starting in June 2014 (DOYs 169176 in 2014). Numerical results are given in Table II after 5 


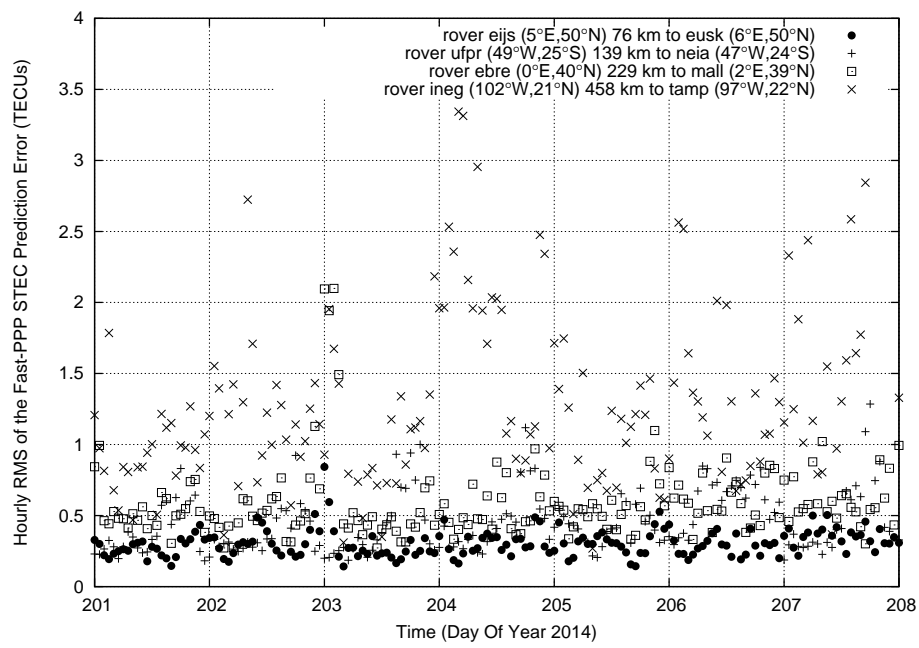

Fig. 12-Hourly RMS of the error of the real-time, two-layer Fast-PPP ionospheric model for some rover locations, located at different distances from the nearest reference station used to compute the model.

and 120 minutes of the navigation filter reset.

The rovers eijs, ufpr and ebre show typical Fast-PPP performances at well-sounded areas. The single-frequency solutions at the beginning of a cold start maintain 30 and 70 centimeters of horizontal and vertical accuracies, respectively. After 30 minutes, errors are reduced to 20 and 40 centimeters, respectively. The final converged values after one hour present 20 and 30 centimeters of horizontal and vertical accuracy, respectively. These figures are several times better than for GRAPHIC positioning (i.e., without using ionospheric corrections). The Fast-PPP dual-frequency solutions reduce the PPP convergence time needed to achieve a navigation error below 1 decimeter, between $60 \%$ to $80 \%$ in the horizontal component and $30 \%$ to $60 \%$ in the vertical component (see Table III).

The effect of increasing the distance to the nearest reference station used to compute the Fast-PPP ionospheric model can be appreciated in the positioning results of rover lkhu, at 455 kilometers. The initial errors in single-frequency Fast-PPP worse to 40 centimeters (horizontal) and meter-level (vertical). The Fast-PPP final HPE and VPE are the same 20 and 30 centimeters of GRAPHIC, still shortening the GRAPHIC convergence time. In dual-frequency, the Fast-PPP solution marginally reduces the PPP convergence time.

In order to illustrate the limits of the Fast-PPP ionospheric corrections, the results of low-latitude rovers ineg and bogt are included. According to Table II, these rovers are located at 458 and 860 kilometers of the nearest reference station, respectively. At the user level, only single-frequency users take advantage of the Fast-PPP ionospheric corrections. However, because the confidence levels (i.e., the sigmas) of the ionospheric corrections are used as weights in the navigation filter, the Fast-PPP dual-frequency solution never is worse than the PPP solutions. Providing a realistic sigma is a key asset of the Fast-PPP technique. This is achieved in the ionospheric filter by adapting the process noise to the latitude and by properly weighting the input measurements in the left hand side of equation (3).

The convergence assessment confirms that the Fast-PPP precise ionospheric modeling added to the navigation filter is translated into the user positioning resulting in a quicker convergence than the ionosphere-free solutions. Moreover, the final accuracy once the filter has converged remains untouched, thanks to the unbiased nature of the Fast-PPP ionosphere. This can be noticed in the position domain, since the FastPPP single-frequency solution converges to the GRAPHIC solution, which is considered as a reference because is free of ionospheric miss-modeling. Finally, the results indicate that a denser network of receivers (or a finer grid) should be used in the equatorial region to keep the ionospheric error at the 1 TECU level, and thus, a similar user domain performance as observed in mid-latitudes.

All cases analyzed in Fig. 13 include the single- and dualfrequency navigation results using the ionospheric corrections from the Rapid GIM from IGS. Clearly, the improvements observed with Fast-PPP ionospheric corrections are not maintained with the corrections computed from the IGS-GIMs. The positioning results using the IGS-GIMs ionosphere incur a deterioration of the classical ionosphere-free solutions. This particularly occurs at rovers located in the equatorial region. The reasons for such deterioration are related to the missmodelings of the IGS-GIMs (seen in the post-fit residuals of the ionospheric test of Fig. 8) and the low RMS values present in the IONosphere map EXchange format (IONEX) files of the IGS-GIMs, which have been used to weight the ionospheric corrections in the navigation filter. This latter effect has been mitigated by means of augmenting the RMS values present in the IGS GIM to similar standard deviations used in the FastPPP ionospheric corrections. This is evidenced in right column of in Fig. 13, where both solutions exhibit similar formal errors in the $3 \mathrm{D}$ positioning.

\section{Actual vs. Formal Error Assessment}

In the previous subsection, it has been shown that the accuracy of the ionospheric corrections is far from homogeneous. Then, it is important to provide the confidence bounds associated to the CPF corrections (i.e., satellite clocks, ionosphere, DCBs). In fact, the margin between the actual and formal errors of Fast-PPP users depends on the degree of realism of (i) the CPF corrections and (ii) the a-priori hypothesis assumed (i.e., standard deviations of pseudoranges and the carrier-phase pre-fit measurements) and the noise of the random walk process used to estimate the wet tropospheric zenith wet delay [35]. The relative weights between corrections and measurements are key for the navigation filter to correctly mix such different sources of information.

This subsection is devoted to assess the consistency at the user domain achieved by the Fast-PPP technique for the entire rover network, including rover receivers located at 800 kilometers of the nearest station used to derive the ionospheric model. HPE and VPE are computed for every epoch within independent batches of 24 hours. Within the day, data are 
processed without resetting the state of the navigation filter. A total of 31 days (DOYs 169-200 in 2014) are merged in every Stanford plot shown in Fig. 14, assessing the horizontal (left column) and vertical (right column) components and the dual-frequency (top row) and single-frequency (bottom row) navigation modes.

It can be seen that 95\% HPE and VPE are respectively 36 and 63 centimeters for single-frequency solutions and the corresponding values for dual-frequency solutions are 11 and 15 centimeters, respectively. It is observed that such figures are achieved with safe margins with respect to the Horizontal Protection Level (HPL) and Vertical Protection Level (VPL). The Protection Levels are computed as $H P L=6.00 \sigma_{H}$ and $V P L=5.33 \sigma_{V}$, where $\sigma_{H}$ and $\sigma_{V}$ are the formal errors computed by the Kalman navigation filter. The values of 6.00 and 5.33 are the $\mathrm{K}$-factors associated with probabilities of the Misleading Information (MI) of $2 \cdot 10^{-9}$ and $10^{-7}$, for the horizontal and vertical component, see Appendix J of [36]. Numerically, 95\% HPL and VPL are respectively 2.08 and 3.34 meters for single-frequency solutions and the corresponding values for dual-frequency solutions are 57 and 87 centimeters.

\section{Conclusions}

An end-to-end performance assessment of the Fast-PPP technique has been presented. The CPF provides a global PPP service thanks to the determination in real-time of precise satellite orbits and clocks on the same order of accuracy that the IGS real-time products; a few centimeters and a few tenths of a nanosecond, respectively. Fast-PPP users are enabled to perform worldwide undifferenced ambiguity fixing because of the estimate of the fractional part of the ambiguities. Once highly accurate satellite orbits, clocks and fractional carrier-phase biases are made available to users in real time, the remaining challenge is the computation of an accurate ionospheric model to enhance performances of both singleand dual-frequency PPP users over well-sounded areas.

A dedicated metric has been introduced to assess the suitability of ionospheric models for high-accuracy navigation. It has been shown that the two-layer, ambiguity-fixed, Fast-PPP ionospheric real-time estimates present a post-fit residuals of a tenth of 1 TECU (RMS) at the reference stations used to derive the model. The error of the real-time model once it is smoothed to provide a global coverage reaches 1 TECU. This error has been shown similar to the accuracy of the Fast-PPP real-time model once it is interpolated at the user location (by independent rovers) at a few hundreds of kilometers, depending on the latitude. Therefore, the Fast-PPP ionosphere can be used in combination with precise orbits and clocks, maintaining their accuracy. This is not the case for the well known IGS-GIMs, which have accuracies that are several times worse. It has been shown that the difference between the two ionospheric models is greater at low latitudes and around the local noon.

Fast-PPP dual-frequency users benefit from the precise ionospheric modeling through a reduction in the convergence time compared to the classic PPP solutions, not only in mid-latitude regions but also under more challenging ionospheric conditions such as those found in the equatorial region. This is noticeable (between $60 \%$ to $80 \%$ in the horizontal component and $30 \%$ to $60 \%$ in the vertical component) for rover baselines up to 500 kilometers, which makes feasible to use a sparse reference station network of about one hundred of stations to derive the Fast-PPP ionospheric model. Larger baseline distances or isolated rovers where the ionospheric prediction is degraded to several TECUs, only show a slight improvement. Thanks to the realism of the confidence levels of the Fast-PPP corrections calculated at the CPF, the navigation accuracy of Fast-PPP is never poorer than the ionosphere-free solutions.

It has been shown how the accuracy of single-frequency users are directly affected by the quality of the ionospheric estimates. The results prove the improvement in the Fast-PPP ionospheric model compared with the IGS-GIMs, particularly at low latitudes. Fast-PPP single-frequency solutions converge to the ionospheric-free GRAPHIC solution, indicating that the Fast-PPP ionospheric corrections are not biased (i.e., no missmodeling) and the associated confidence bounds are realistic. Finally, it has been shown that the navigation of receivers located up to 800 kilometers to the nearest station used to derive the Fast-PPP ionospheric model is safely bounded by protection levels of few meters for single-frequency receivers (mass-market) and some decimeters for dual-frequency receivers.

\section{ACKNOWLEDGMENTS}

The authors acknowledge the use of data from the International GNSS Service and the Centre National d'Études Spatiales. This work has been partially sponsored by the European Space Agency together with the industrial partner FUGRO. The Technical University of Catalonia contributed with an FPI-UPC grant. We also would like to thank to the Spanish Ministry of Science and Innovation project CGL201566410-P and the GNAVIS project from EC-FP7 Grant Nr. 287203. The activity has been developed for the ESA funded project ICASES.

\section{REFERENCES}

[1] J. F. Zumberge, M. B. Heflin, D. C. Jefferson, M. M. Watkins, and F. H. Webb, "Precise Point Positioning for the efficient and robust analysis of GPS data from large networks," Journal of Geophysical Research: Solid Earth, vol. 102, no. B3, pp. 5005-5017, 1997. [Online]. Available: http://dx.doi.org/10.1029/96JB03860

[2] J. Kouba and P. Héroux, "Precise Point Positioning Using IGS Orbit and Clock Products," GPS Solutions, vol. 5, no. 2, pp. 12-28, 2001. [Online]. Available: http://dx.doi.org/10.1007/PL00012883

[3] G. Beutler, M. Rothacher, S. Schaer, T. Springer, J. Kouba, and R. Neilan, "The International GPS Service (IGS): An interdisciplinary service in support of Earth sciences." Advances in Space Research, vol. 23, no. 4, pp. 631-653, 1999. [Online]. Available: http: //dx.doi.org/10.1016/S0273-1177(99)00160-X

[4] B. W. Remondi, "Performing centimetre Accuracy Relative Surveys in Seconds Using GPS Carrier Phase," in First International Symposium on Precise Positioning with the Global Positioning System, vol. 2, Rockville, Maryland, apr 1985, pp. 789-798. [Online]. Available: https://archive.org/details/positioningwith00inte 
[5] Colombo, O.L., Hernández-Pajares, M., Juan, M., and Sanz, J., "Wide-Area carrier-phase ambiguity resolution using a tomographic model of the Ionosphere." Navigation, vol. 49, no. 1, pp. 61-69, 2002. [Online]. Available: http://www.ion.org/publications/abstract.cfm?jp= j\&articleID=2323

[6] D. Laurichesse and F. Mercier, "Integer Ambiguity Resolution on Undifferenced GPS Phase Measurements and Its Application to PPP.” in Proceedings of the 20th International Technical Meeting of the Satellite Division, Institute of Navigation, Fort Worth, Texas (USA), Sep. 2007, pp. 839 - 848. [Online]. Available: http://www.ion.org/publications/abstract.cfm?articleID $=7584$

[7] P. Collins, F. Lahaye, P. Heroux, and S. Bisnath, "Precise Point Positioning with Ambiguity Resolution using the Decoupled Clock Model," in Proceedings of the 21st International Technical Meeting of the Satellite Division of The Institute of Navigation (ION GNSS 2008), Savannah, GA, USA, Sep. 2008, pp. 1315-1322. [Online]. Available: https://www.ion.org/publications/abstract.cfm?articleID=8043

[8] M. Ge, M. Gendt, M. Rothacher, C. Shi, and J. Liu, "Resolution of GPS Carrier-Phase Ambiguities in Precise Point Positioning (PPP) with Daily Observations." Journal of Geodesy, vol. 82, no. 7, pp. 389-399, 2008. [Online]. Available: http://dx.doi.org/10.1007/s00190-007-0187-4

[9] L. Mervart, Z. Lukes, C. Rocken, and T. Iwabuchi, "Precise Point Positioning with Ambiguity Resolution in Real-Time," in Proceedings of the 21st International Technical Meeting of the Satellite Division of The Institute of Navigation (ION GNSS 2008), Savannah, GA, USA, Sep. 2008, pp. 397-405. [Online]. Available: https://www.ion.org/publications/abstract.cfm?articleID=7969

[10] J. Shi and Y. Gao, "A comparison of three ppp integer ambiguity resolution methods," GPS Solutions, vol. 18, no. 4, pp. 519-528, 2014 [Online]. Available: http://dx.doi.org/10.1007/s10291-013-0348-2

[11] P. J. G. Teunissen and A. Khodabandeh, "Review and principles of PPPRTK methods," Journal of Geodesy, vol. 89, no. 3, pp. 217-240, 2014 [Online]. Available: http://dx.doi.org/10.1007/s00190-014-0771-3

[12] J. Juan, M. Hernández-Pajares, J. Sanz, P. Ramos-Bosch, A. AragonAngel, R. Orús, W. Ochieng, S. Feng, P. Coutinho, J. Samson, and M. Tossaint, "Enhanced Precise Point Positioning for GNSS Users." IEEE Transactions on Geoscience and Remote Sensing, 2012. [Online]. Available: http://dx.doi.org/10.1109/TGRS.2012.2189888

[13] M. Hernández-Pajares, J. Juan, J. Sanz, J. Samson, and M. Tossaint, "Method, Apparatus and System for Determining a Position of an Object Having a Global Navigation Satellite System Receiver by Processing Undifferenced Data Like Carrier Phase Measurements and External Products Like Ionosphere Data. international patent application PCT/EP2011/001512 (ESA ref.: ESA/PAT/566)." 2011. [Online]. Available: https://patentscope.wipo.int/search/en/WO2012130252

[14] A. Rovira-Garcia, J. Juan, J. Sanz, and G. González-Casado, "A Worldwide Ionospheric Model for Fast Precise Point Positioning," Geoscience and Remote Sensing, IEEE Transactions on, vol. 53, no. 8, pp. 4596-4604, Aug 2015. [Online]. Available: http: //ieeexplore.ieee.org/xpl/articleDetails.jsp?arnumber $=7053952$

[15] ESA, "ICASES: Ionospheric Conditions and Associated Scenarios for EGNOS Selected from the last Solar Cycle. PO1520026618/01." Noordwick, The Netherlands, 2014.

[16] A. Rovira-Garcia, J. Juan, J. Sanz, G. González-Casado, and D. Ibáñez Segura, "Accuracy of Ionospheric Models used in GNSS and SBAS: Methodology and Analysis," Journal of Geodesy, vol. 90, no. 3, pp. 229-240, Oct 2015. [Online]. Available: http://dx.doi.org/10.1007/s00190-015-0868-3

[17] J. Klobuchar, "Ionospheric Time-Delay Algorithm for Single-Frequency GPS Users," Aerospace and Electronic Systems, IEEE Transactions on, vol. AES-23, no. 3, pp. 325-331, May 1987. [Online]. Available: http://dx.doi.org/10.1109/TAES.1987.310829

[18] G. Di Giovanni and S. Radicella, "An Analytical Model of the Electron Density Profile in the Ionosphere." Advances in Space Research, vol. 10, no. 11, pp. 27-30, 1990. [Online]. Available: http://dx.doi.org/10.1016/0273-1177(90)90301-F
[19] M. Hernández-Pajares, J. Juan, J. Sanz, R. Orús, A. Garcia-Rigo, J. Feltens, A. Komjathy, S. Schaer, and A. Krankowski, "The IGS VTEC maps: a reliable source of ionospheric information since 1998," Journal of Geodesy, vol. 83, no. 3-4, pp. 263-275, 2009. [Online]. Available: http://dx.doi.org/10.1007/s00190-008-0266-1

[20] J. Sanz, J. Juan, G. González-Casado, R. Prieto-Cerdeira, S. S., and R. Orús, "Novel Ionospheric Activity Indicator Specifically Tailored for GNSS Users," in Proceedings of ION GNSS+ 2014, Tampa, Florida (USA), Sep. 2014, pp. 1173-1182. [Online]. Available: http://www.ion.org/publications/abstract.cfm?jp=p\&articleID=12269

[21] J. Juan, A. Rius, M. Hernández-Pajares, and J. Sanz, "A two-layer model of the ionosphere using Global Positioning System data," Geophysical Research Letters, vol. 24, no. 4, pp. 393-396, 1997. [Online]. Available: http://dx.doi.org/10.1029/97GL00092

[22] G. Wubbena, A. Bagge, G. Seeber, V. Boder, and P. Hankemeier, "Reducing Distance Dependent Errors for Real Time Precise DGPS Applications by Establishing Reference Station Networks." in Proceedings of ION GPS 1996, Kansas City, Missouri (USA), 1996, pp. 1845-1852. [Online]. Available: http://www.ion.org/publications/ abstract.cfm?articleID $=2722$

[23] S. Datta-Barua, T. Walter, E. Altshuler, J. Blanch, and P. Enge, "Dst as an Indicator of Potential Threats to WAAS Integrity and Availability," in Proceedings of ION GPS 2005, USA, Sep. 2005, pp. 2365-2373. [Online]. Available: http://www.ion.org/publications/ abstract.cfm? articleID $=6442$

[24] C. A. Loewe and G. W. Prölss, "Classification and mean behavior of magnetic storms," Journal of Geophysical Research: Space Physics, vol. 102, no. A7, pp. 14209-14213, 1997. [Online]. Available: http://dx.doi.org/10.1029/96JA04020

[25] J. Sanz, J. Juan, and M. Hernández-Pajares, GNSS Data Processing, Vol. I: Fundamentals and Algorithms. Noordwijk, the Netherlands: ESA Communications, ESTEC TM-23/1, 2013. [Online]. Available: http:// www.navipedia.net/GNSS_Book/ESA_GNSS-Book_TM-23_Vol_I.pdf

[26] International GNSS Service Products, Jan. 2014. [Online]. Available: http://www.igs.org/products/data

[27] IGS Real Time Pilot Project, "http://www.rtigs.net," Jan. 2014. [Online]. Available: http://www.rtigs.net

[28] K. Rawer, Propagation of Decameter Waves (HF-band) in Meteorological and Astronomical Influences on Radio Wave Propagation. New York: Ed. Landmark, B. Pergamon Press, 1963.

[29] A. J. Mannucci, B. D. Wilson, D. N. Yuan, C. H. Ho, U. J. Lindqwister, and T. F. Runge, "A global mapping technique for GPS-derived ionospheric total electron content measurements," Radio Science, vol. 33, no. 3, pp. 565-582, 1998. [Online]. Available: http://dx.doi.org/10.1029/97RS02707

[30] G. González-Casado, J. M. Juan, J. Sanz, A. Rovira-Garcia, and A. Aragon-Angel, "Ionospheric and plasmaspheric electron contents inferred from radio occultations and global ionospheric maps," Journal of Geophysical Research: Space Physics, vol. 120, no. 7, pp. 5983-5997, 2015. [Online]. Available: http: //dx.doi.org/10.1002/2014JA020807

[31] M. Juan, J. Sanz, G. González-Casado, A. Rovira-Garcia, D. Ibáñez Segura, R. Orús-Pérez, R. Prieto-Cerdeira, and S. Schlueter, "Accurate reference ionospheric model for testing GNSS ionospheric correction in EGNOS and Galileo," in Proceedings of the 7th ESA Workshop on Satellite Navigation Technologies: Era of Galileo IOV (NAVITEC 2014), ESA/ESTEC, Noordwijk, The Netherlands, Dec. 2014, pp. 1-8. [Online]. Available: http://gage6.upc.es/gAGE_WEB/papers/2014/ 73415_Juan.pdf

[32] P. J. G. Teunissen, "The geometry-free GPS ambiguity search space with a weighted ionosphere," Journal of Geodesy, vol. 71, no. 6, pp. 370-383, 1997. [Online]. Available: http://dx.doi.org/10.1007/ s001900050105

[33] T. P. Yunck, "Coping with the Atmosphere and Ionosphere in Precise Satellite and Ground Positioning," Environmental Effects on Spacecraft 
Positioning and Trajectories, vol. 73, pp. 1-16, 1993. [Online]. Available: http://dx.doi.org/10.1029/GM073p0001

[34] Stanford GPS Laboratory, "WAAS Precision Approach Metrics Accuracy, Integrity, Continuity and Availability," 1997. [Online]. Available: http://waas.stanford.edu/metrics.html

[35] M. Hernández-Pajares, J. M. Juan, J. Sanz, O. L. Colombo, and H. van der Marel, "A new strategy for real-time integrated water vapor determination in wadgps networks," Geophysical Research Letters, vol. 28, no. 17, pp. 3267-3270, 2001. [Online]. Available: http://dx.doi.org/10.1029/2001GL012930

[36] RTCA, "Minimum Operational Performance Standards for Global Positioning System/Wide Area Augmentation System Airborne Equipment. RTCA Document 229-C," Washington, USA, 2006. 
TABle II. Horizontal and Vertical Positioning Errors together with the Formal 3D ERror At 5 AND 120 MinUtes AFTER the NAVIGATION FILTER IS RESET FOR SINGLE- AND DUAL-FREQUENCY NAVIGATION MODES (RESULTS IN METERS).

\begin{tabular}{|c|c|c|c|c|c|c|c|c|c|c|}
\hline \multirow{2}{*}{ rover } & \multirow{2}{*}{$\begin{array}{l}\text { geomagnetic } \\
\text { coordinates }\end{array}$} & \multirow{2}{*}{ distance } & \multirow{2}{*}{\multicolumn{2}{|c|}{$\begin{array}{l}\text { processing } \\
\text { mode }\end{array}$}} & \multicolumn{3}{|c|}{ results after 5 minutes } & \multicolumn{3}{|c|}{ results after 120 minutes } \\
\hline & & & & & HPE & VPE & $\sigma_{3 D}$ & HPE & VPE & $\sigma_{3 D}$ \\
\hline \multirow{6}{*}{ eijs } & \multirow{6}{*}{$52^{\circ} \mathrm{E} 52^{\circ} \mathrm{N}$} & \multirow{6}{*}{$77 \mathrm{~km}$} & \multirow{3}{*}{$1 \mathrm{~F}$} & GRAPHIC & 8.95 & 5.52 & 22.61 & 0.25 & 0.26 & 0.63 \\
\hline & & & & Fast-PPP & 0.18 & 0.47 & 0.73 & 0.11 & 0.17 & 0.34 \\
\hline & & & & IGS-GIM & 5.04 & 3.84 & 11.89 & 0.52 & 0.58 & 0.49 \\
\hline & & & \multirow{3}{*}{$2 \mathrm{~F}$} & PPP & 0.31 & 0.52 & 0.91 & 0.07 & 0.07 & 0.12 \\
\hline & & & & Fast-PPP & 0.13 & 0.30 & 0.42 & 0.05 & 0.06 & 0.10 \\
\hline & & & & IGS-GIM & 0.63 & 1.38 & 0.77 & 0.16 & 0.15 & 0.11 \\
\hline \multirow{6}{*}{ ufpr } & \multirow{6}{*}{$-15^{\circ} \mathrm{E}-15^{\circ} \mathrm{N}$} & \multirow{6}{*}{$140 \mathrm{~km}$} & \multirow{3}{*}{$1 \mathrm{~F}$} & GRAPHIC & 10.03 & 8.28 & 23.04 & 0.30 & 0.41 & 0.69 \\
\hline & & & & Fast-PPP & 0.24 & 0.70 & 1.32 & 0.19 & 0.29 & 0.51 \\
\hline & & & & IGS-GIM & 2.76 & 4.64 & 5.60 & 0.69 & 1.23 & 0.46 \\
\hline & & & \multirow{3}{*}{$2 \mathrm{~F}$} & PPP & 0.34 & 0.63 & 1.09 & 0.06 & 0.05 & 0.13 \\
\hline & & & & Fast-PPP & 0.20 & 0.43 & 0.84 & 0.06 & 0.05 & 0.12 \\
\hline & & & & IGS-GIM & 0.94 & 2.82 & 0.81 & 0.39 & 0.42 & 0.12 \\
\hline \multirow{6}{*}{ ebre } & \multirow{6}{*}{$43^{\circ} \mathrm{E} 43^{\circ} \mathrm{N}$} & \multirow{6}{*}{$230 \mathrm{~km}$} & \multirow{3}{*}{$1 \mathrm{~F}$} & GRAPHIC & 8.06 & 7.52 & 32.69 & 0.35 & 0.33 & 1.43 \\
\hline & & & & Fast-PPP & 0.26 & 0.55 & 1.07 & 0.27 & 0.30 & 1.35 \\
\hline & & & & IGS-GIM & 4.87 & 4.11 & 16.41 & 0.55 & 0.61 & 0.55 \\
\hline & & & \multirow{3}{*}{$2 \mathrm{~F}$} & PPP & 0.37 & 0.87 & 1.05 & 0.08 & 0.13 & 0.14 \\
\hline & & & & Fast-PPP & 0.20 & 0.39 & 0.66 & 0.08 & 0.11 & 0.12 \\
\hline & & & & IGS-GIM & 0.73 & 1.44 & 0.90 & 0.23 & 0.17 & 0.13 \\
\hline \multirow{6}{*}{ lkhu } & \multirow{6}{*}{$40^{\circ} \mathrm{E} 40^{\circ} \mathrm{N}$} & \multirow{6}{*}{$455 \mathrm{~km}$} & \multirow{3}{*}{$1 \mathrm{~F}$} & GRAPHIC & 11.19 & 9.65 & 25.93 & 0.32 & 0.41 & 0.71 \\
\hline & & & & Fast-PPP & 0.41 & 0.92 & 1.67 & 0.23 & 0.36 & 0.52 \\
\hline & & & & IGS-GIM & 2.79 & 3.78 & 6.50 & 0.53 & 1.20 & 0.51 \\
\hline & & & & PPP & 0.45 & 0.92 & 1.12 & 0.11 & 0.10 & 0.13 \\
\hline & & & $2 \mathrm{~F}$ & Fast-PPP & 0.35 & 0.67 & 0.96 & 0.09 & 0.09 & 0.12 \\
\hline & & & & IGS-GIM & 0.70 & 1.98 & 0.93 & 0.20 & 0.33 & 0.12 \\
\hline & & & & GRAPHIC & 2.16 & 1.79 & 25.29 & 0.23 & 0.21 & 0.77 \\
\hline & & & $1 \mathrm{~F}$ & Fast-PPP & 0.56 & 0.86 & 2.49 & 0.19 & 0.25 & 0.57 \\
\hline ineo & $31^{\circ} \mathrm{F} 31^{\circ} \mathrm{N}$ & $458 \mathrm{~km}$ & & IGS-GIM & 1.73 & 2.96 & 8.57 & 0.86 & 1.42 & 0.56 \\
\hline & & & & PPP & 0.24 & 0.91 & 1.23 & 0.06 & 0.11 & 0.38 \\
\hline & & & $2 \mathrm{~F}$ & Fast-PPP & 0.24 & 0.79 & 1.14 & 0.06 & 0.12 & 0.37 \\
\hline & & & & IGS-GIM & 0.94 & 1.72 & 1.10 & 0.43 & 0.40 & 0.30 \\
\hline & & & & GRAPHIC & 19.36 & 10.59 & 35.61 & 1.38 & 0.84 & 0.94 \\
\hline & & & $1 \mathrm{~F}$ & Fast-PPP & 1.04 & 2.04 & 3.37 & 0.44 & 0.62 & 0.66 \\
\hline boot & $3^{\circ} \mathrm{F} 16^{\circ} \mathrm{N}$ & $860 \mathrm{~km}$ & & IGS-GIM & 5.42 & 4.53 & 9.44 & 0.96 & 1.04 & 0.61 \\
\hline Dogl & $3 \mathrm{E} 10 \mathrm{~N}$ & $000 \mathrm{~km}$ & & PPP & 0.49 & 0.94 & 1.38 & 0.07 & 0.06 & 0.16 \\
\hline & & & $2 \mathrm{~F}$ & Fast-PPP & 0.48 & 0.91 & 1.33 & 0.08 & 0.07 & 0.15 \\
\hline & & & & IGS-GIM & 0.96 & 2.60 & 1.19 & 0.41 & 0.25 & 0.15 \\
\hline
\end{tabular}




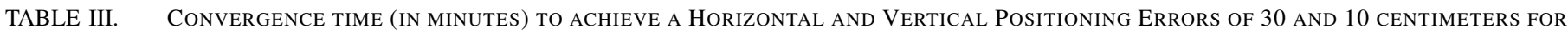
SINGLE- AND DUAL-FREQUENCY NAVIGATION MODES, RESPECTIVELY. SOLUTIONS NOT REACHING SUCH ACCURACIES WITHIN 120 MINUTES ARE SHOWN WITH A HYPHEN.

\begin{tabular}{|c|c|c|c|c|c|c|c|c|c|c|c|c|c|c|}
\hline \multirow{3}{*}{ rover } & \multirow{3}{*}{$\begin{array}{l}\text { geomagnetic } \\
\text { coordinates }\end{array}$} & \multirow{3}{*}{ distance } & \multicolumn{6}{|c|}{ Single Frequency } & \multicolumn{6}{|c|}{ Dual Frequency } \\
\hline & & & \multicolumn{2}{|c|}{ GRAPHIC } & \multicolumn{2}{|c|}{ Fast-PPP } & \multicolumn{2}{|c|}{ IGS-GIM } & \multicolumn{2}{|c|}{ PPP } & \multicolumn{2}{|c|}{ Fast-PPP } & \multicolumn{2}{|c|}{ IGS-GIM } \\
\hline & & & $\mathrm{H}$ & $\mathrm{V}$ & $\mathrm{H}$ & $\mathrm{V}$ & $\mathrm{H}$ & $\mathrm{V}$ & $\mathrm{H}$ & $\mathrm{V}$ & $\mathrm{H}$ & $\mathrm{V}$ & $\mathrm{H}$ & $\mathrm{V}$ \\
\hline eijs & $52^{\circ} \mathrm{E} 52^{\circ} \mathrm{N}$ & $77 \mathrm{~km}$ & 82 & 77 & 0 & 20 & - & - & 78 & 60 & 8 & 26 & - & - \\
\hline ufpr & $-15^{\circ} \mathrm{E}-15^{\circ} \mathrm{N}$ & $140 \mathrm{~km}$ & 114 & - & 4 & 72 & - & - & 57 & 69 & 22 & 46 & - & - \\
\hline ebre & $43^{\circ} \mathrm{E} 43^{\circ} \mathrm{N}$ & $230 \mathrm{~km}$ & 100 & 100 & 1 & 44 & - & - & 97 & - & 40 & 80 & - & - \\
\hline lkhu & $40^{\circ} \mathrm{E} 40^{\circ} \mathrm{N}$ & $455 \mathrm{~km}$ & 118 & - & 37 & - & - & - & - & 103 & 100 & 98 & - & - \\
\hline ineg & $31^{\circ} \mathrm{E} 31^{\circ} \mathrm{N}$ & $458 \mathrm{~km}$ & 74 & 82 & 50 & 60 & - & - & 53 & 105 & 54 & 106 & - & - \\
\hline bogt & $3^{\circ} \mathrm{E} 16^{\circ} \mathrm{N}$ & $860 \mathrm{~km}$ & - & - & - & - & - & - & 84 & 69 & 92 & 66 & - & - \\
\hline
\end{tabular}



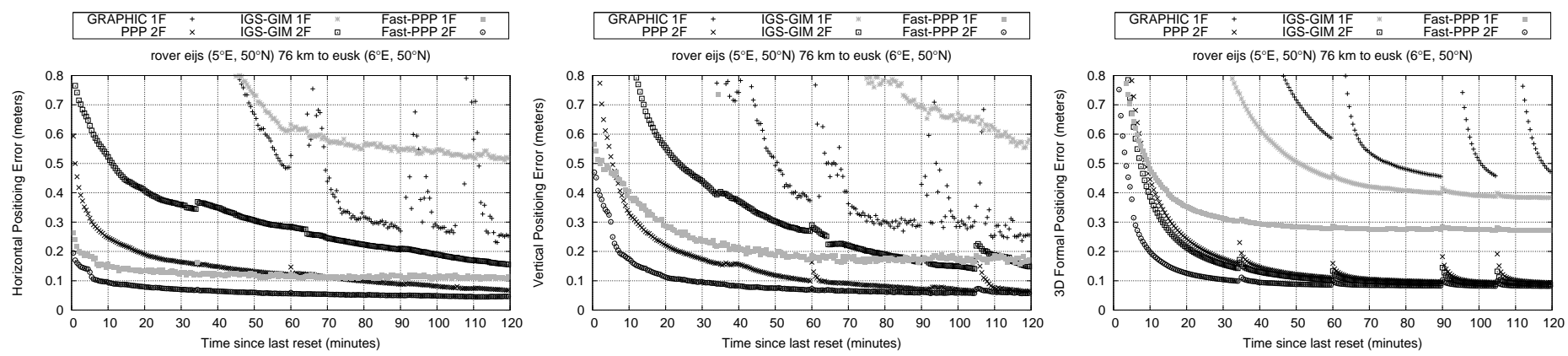
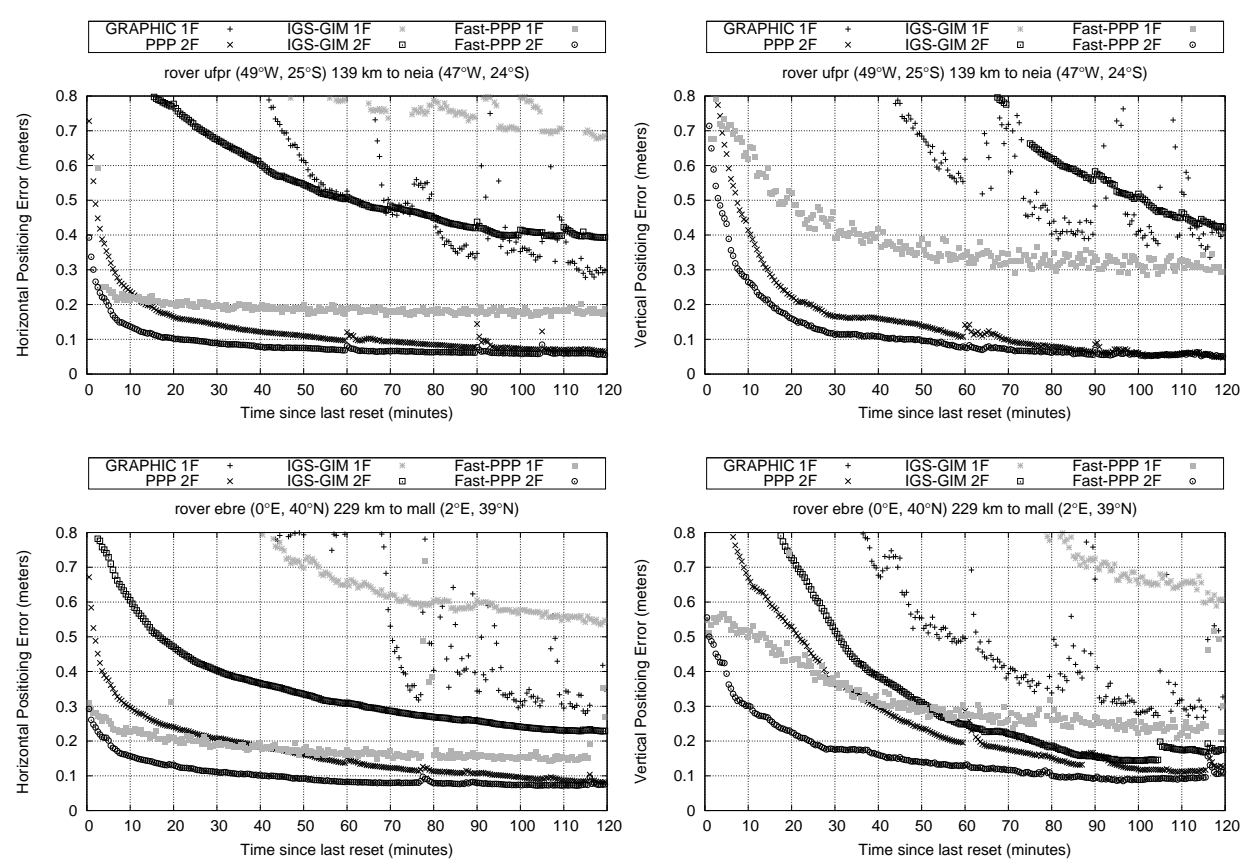

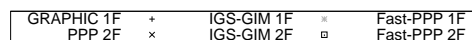
rover Ikhu $\left(95^{\circ} \mathrm{W}, 29^{\circ} \mathrm{N}\right) 455 \mathrm{~km}$ to hamm $\left(90^{\circ} \mathrm{W}, 30^{\circ} \mathrm{N}\right)$

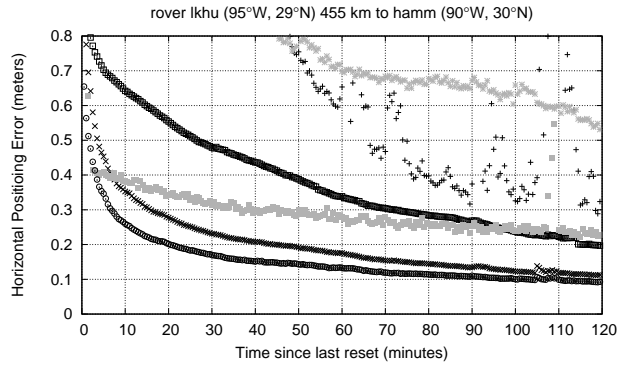

GRAPHIC $1 F$

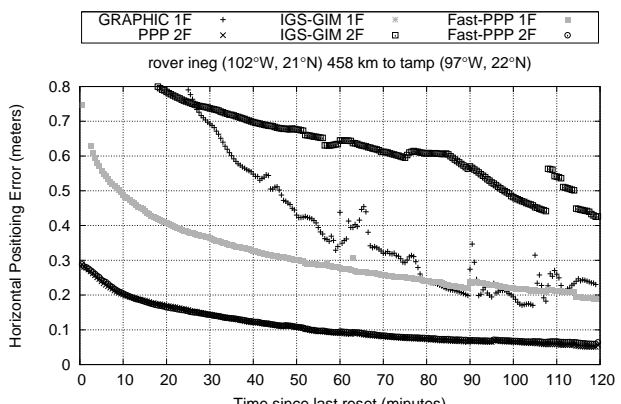

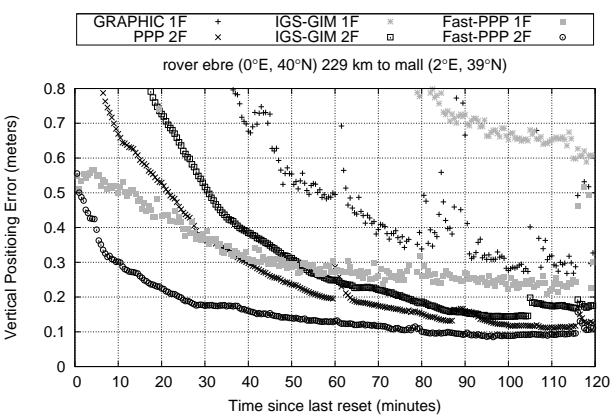

rover ebre $\left(0^{\circ} \mathrm{E}, 40^{\circ} \mathrm{N}\right) 229 \mathrm{~km}$ to mall $\left(2^{\circ} \mathrm{E}, 39^{\circ} \mathrm{N}\right)$
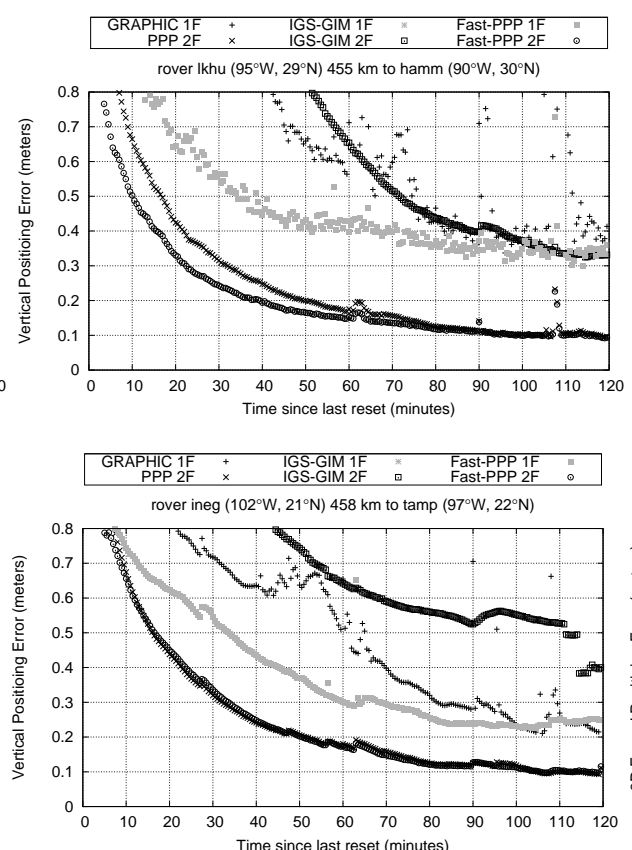

\begin{tabular}{|rllll}
\hline GRAPHIC 1F & + & IGS-GIM 1F & $*$ & Fast-PPP 1F \\
PPP 2F & $\times$ & IGS-GIM 2F & a & Fast-PPP 2F \\
\hline
\end{tabular}

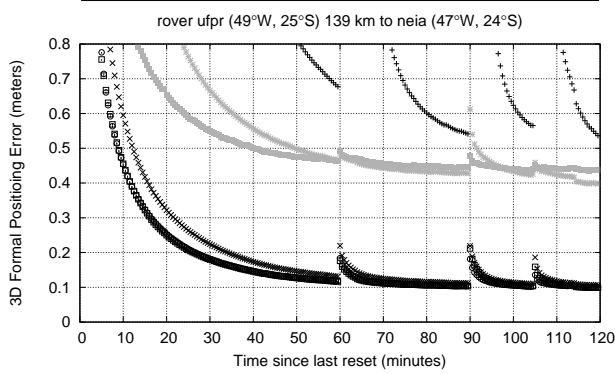

\begin{tabular}{rllll}
\hline GRAPHIC 1F & + & IGS-GIM 1F & $*$ & Fast-PPP 1F \\
PPP 2F & $\times$ & IGS-GIM 2F & ( & Fast-PPP 2F \\
\hline
\end{tabular}

rover ebre $\left(0^{\circ} \mathrm{E}, 40^{\circ} \mathrm{N}\right) 229 \mathrm{~km}$ to mall $\left(2^{\circ} \mathrm{E}, 39^{\circ} \mathrm{N}\right)$

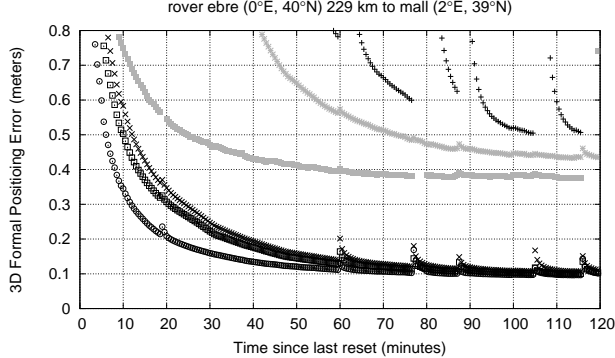

\begin{tabular}{|lllll}
\hline GRAPHIC 1F & + & IGS-GIM 1F & $*$ & Fast-PPP 1F \\
PPP 2F & $\times$ & IGS-GIM 2F & F & Fast-PPP 2F \\
\hline
\end{tabular}

rover lkhu $\left(95^{\circ} \mathrm{W}, 29^{\circ} \mathrm{N}\right) 455 \mathrm{~km}$ to hamm $\left(90^{\circ} \mathrm{W}, 30^{\circ} \mathrm{N}\right)$

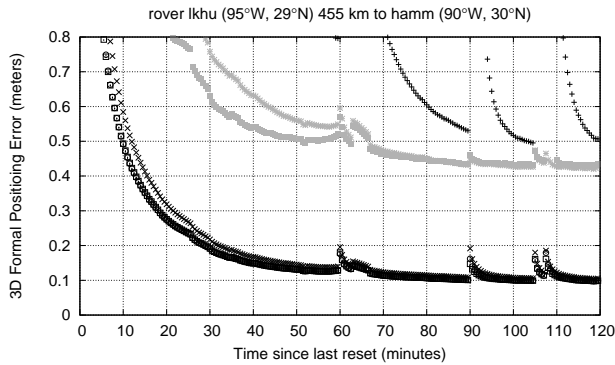

\begin{tabular}{|llll}
\hline GRAPHIC 1F & + & IGS-GIM 1F & \\
PPP 2F & IGS-GIM 2F & Fast-PPP 1F \\
\hline
\end{tabular} rover ineg $\left(102^{\circ} \mathrm{W}, 21^{\circ} \mathrm{N}\right) 458 \mathrm{~km}$ to tamp $\left(97^{\circ} \mathrm{W}, 22^{\circ} \mathrm{N}\right)$

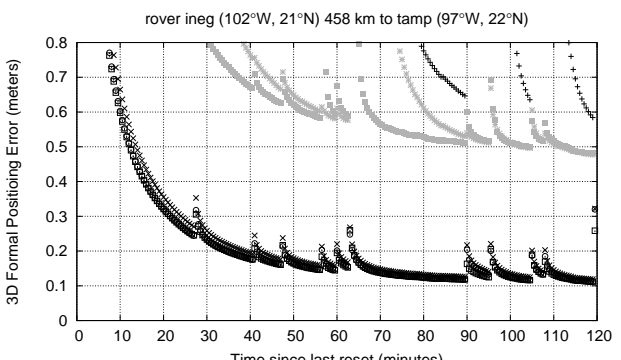

Fig. 13-Horizontal (left) and vertical (center) RMS accuracies as a function of time since the receiver is reset every 2 hours, DOYs 169-200 in 2014. The right column shows the formal error of the 3D positioning. The single and dual-frequency ionosphere-free solutions (GRAPHIC and Classic PPP) are compared with the enhanced positioning using ionosphere data from the Fast-PPP and IGS GIMs. Each mode is identified by GRAPHIC (pluses), Classic PPP (crosses), Fast-PPP single- and dual-frequency (grey squares and circles), IGS-GIMs in IONEX format single- and dual-frequency (grey asterisks and squares). 
All Rovers Fast-PPP 2-Freq [N=904569, 30s]

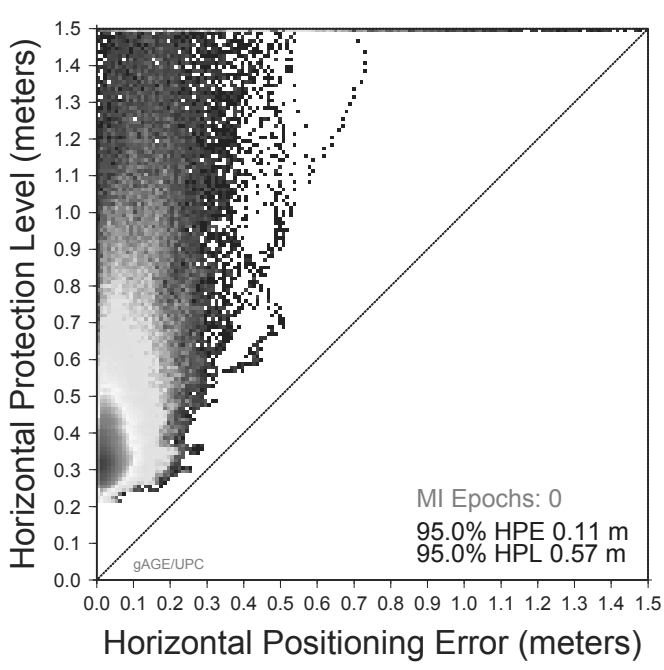

All Rovers Fast-PPP 1-Freq [N=904569, 30s]

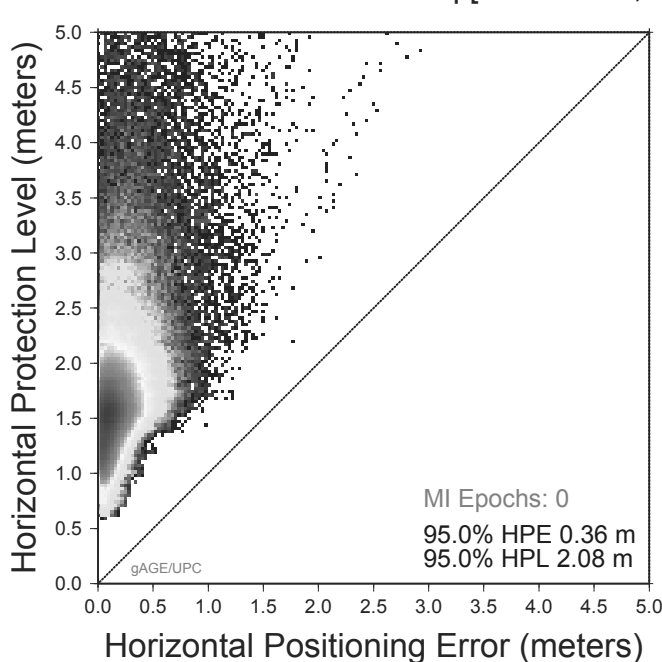

All Rovers Fast-PPP 2-Freq [N=904569, 30s]

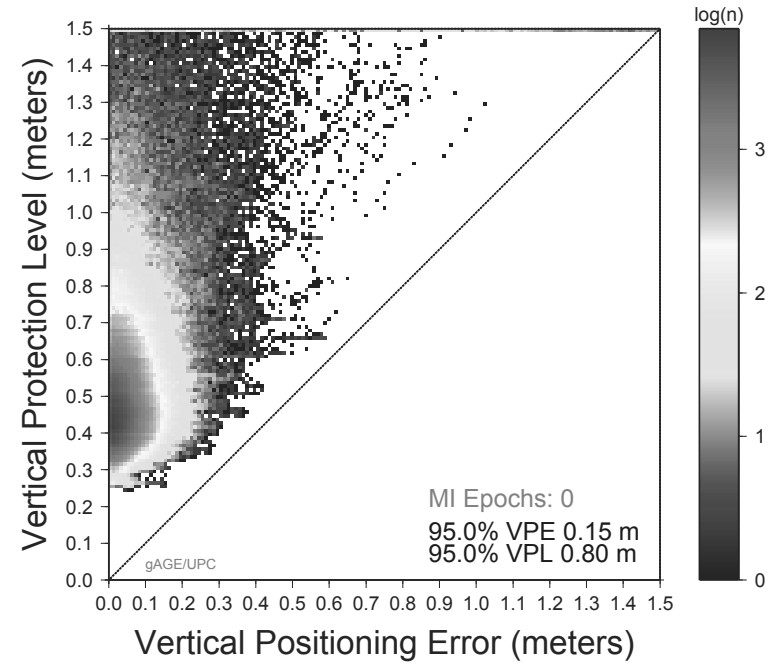

All Rovers Fast-PPP 1-Freq [N=904569, 30s]

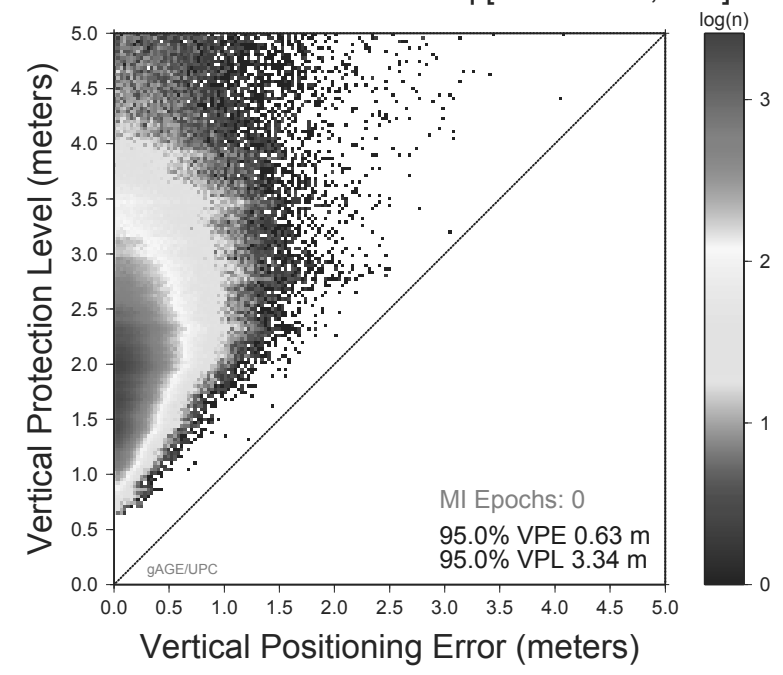

Fig. 14-Horizontal (left column) and vertical (right column) Stanford plots for dual-frequency (top row) and single-frequency (bottom row) Fast-PPP navigation solutions. Horizontal and Vertical Positioning Errors are bounded by the corresponding Horizontal and Vertical Protection Levels. Each plot shows a total of 904569 epochs, merging the navigation solutions of the 15 rovers for DOYs 169-200 in 2014, with a sampling rate of 30 seconds. 